\title{
Gauge Theories and Macdonald Polynomials
}

\author{
Abhijit Gadde ${ }^{\natural}$, Leonardo Rastelli*, Shlomo S. Razamat ${ }^{\star} \diamond$, and Wenbin Yan* \\ * C.N. Yang Institute for Theoretical Physics, Stony Brook, NY 11794-3840, USA \\ ${ }^{\circ}$ California Institute of Technology, Pasadena, CA 91125, USA \\ $\diamond$ Institute for Advanced Study, Princeton, NJ 08540, USA
}

\begin{abstract}
We study the $\mathcal{N}=2$ four-dimensional superconformal index in various interesting limits, such that only states annihilated by more than one supercharge contribute. Extrapolating from the $S U(2)$ generalized quivers, which have a Lagrangian description, we conjecture explicit formulae for all $A$-type quivers of class $\mathcal{S}$, which in general do not have one. We test our proposals against several expected dualities. The index can always be interpreted as a correlator in a two-dimensional topological theory, which we identify in each limit as a certain deformation of two-dimensional Yang-Mills theory. The structure constants of the topological algebra are diagonal in the basis of Macdonald polynomials of the holonomies.
\end{abstract}

KEYwords: SCFT, TQFT, S-duality. 


\section{Contents}

1. Introduction $\quad 2$

2. The $\mathcal{N}=2$ superconformal index $\quad 5$

$\begin{array}{ll}\text { 3. TQFT structure of the index } & 8\end{array}$

4. Limits of the index with additional supersymmetry $\quad 11$

5. Hall-Littlewood index 15

$\begin{array}{lr}\text { 6. Schur index } & 28\end{array}$

$\begin{array}{ll}\text { 7. Macdonald index } & 30\end{array}$

8. Coulomb-branch index 34

$\begin{array}{ll}9 . & \text { Discussion }\end{array}$

A. Construction of the diagonal expression for the $S U(2)$ HL index $\quad 40$

B. Index of short multiplets of $\mathcal{N}=2$ superconformal algebra 44

C. Large $k$ limit of the genus $\mathfrak{g}$ HL index $\quad 48$

D. The unrefined HL index of $T_{4} \quad 49$

E. Proof of the $S U(2)$ Schur index identity 50 


\section{In memory of Francis A. Dolan}

\section{Introduction}

In recent years we have learnt many surprising facts about four-dimensional superconformal field theories (SCFTs), a subject where disparate strands of mathematical physics come together in new beautiful ways. For maximal supersymmetry there is an extraordinarily rich model, $\mathcal{N}=4$ super Yang-Mills, which is a unique theory given a choice of gauge group. Theories with $\mathcal{N}=2$ superconformal symmetry are even richer. The vast majority of them do not have a weakly-coupled regime nor a conventional Lagrangian description. This fact, which may have been suspected since the early days of string dualities, has taken center stage after the more explicit construction of the $\mathcal{N}=2$ superconformal theories of "class $\mathcal{S}$ " [1, 2], most of which are not Lagrangian. ${ }^{1}$

Class $\mathcal{S}$ theories arise by compactification of the six-dimensional $(2,0)$ theory on a punctured Riemann surface $\mathcal{C}$. There is a growing dictionary relating four-dimensional quantities with quantities associated to the surface $\mathcal{C}$. A basic entry of the dictionary identifies the exactly marginal couplings of the $4 d$ theory with the complex structure moduli of $\mathcal{C}{ }^{2}$ According to the celebrated AGT conjecture [7, 8, 9], the $4 d$ partition functions on the $\Omega$-background [10] and on $S^{4}$ [11] are computed by Liouville/Toda theory on $\mathcal{C}$. An analogous relation exists between the $4 d$ superconformal index $[12,13]$ (which can also be viewed as a supersymmetric partition function on $S^{3} \times S^{1}$ ) and topological quantum field theory (TQFT) on $\mathcal{C}[14,15,16]$. In this paper we continue to explore this last relation.

The superconformal index is a simpler observable than the $S^{4}$ partition function, and it should be a good starting point for a microscopic derivation of the $4 d / 2 d$ dictionary from the $6 d(2,0)$ theory. Being coupling-independent, the index is computed by a topological correlator on $\mathcal{C}$ [14], as opposed to a CFT correlator as in the AGT correspondence. For the subset of class $\mathcal{S}$ theories that have a Lagrangian description, it can be easily evaluated in the free-field limit, unlike the $S^{4}$ partition function, which is sensitive to non-perturbative physics and requires a sophisticated localization calculation $[10,11]$.

Despite these simplifying features, the index of class $\mathcal{S}$ theories is still a very non-trivial observable with remarkable mathematical structure. First of all, there is no direct way to compute it for the non-Lagrangian SCFTs, which by definition are not continuously connected

\footnotetext{
${ }^{1}$ Though very large, class $\mathcal{S}$ does not cover the full space of $\mathcal{N}=2$ SCFTs. Counterexamples can be found e.g. in $[1,3]$. See $[4,3,5]$ for the beginning of a classification program for $\mathcal{N}=24 d$ SCFTs.

${ }^{2}$ On the other hand, the conformal factor of the metric on $\mathcal{C}$ is irrelevant (in the RG sense) and its memory lost in the IR SCFT. See [6] for a recent holographic check of this fact.
} 
to free-field theories. ${ }^{3}$ An indirect route is to use the generalized S-dualities [26, 1] that relate non-Lagrangian with Lagrangian theories. This is the strategy used in [15] to evaluate the index of the strongly-coupled SCFT with $E_{6}$ flavor symmetry [27]. In principle this procedure could be carried out recursively to find the index of all the non-Lagrangian theories, but it suffers from two drawbacks: conceptually, one would rather use the index to test dualities, than assume dualities to compute the index; and practically, this program gets quickly too complicated to be useful.

What one should aim for is a direct algorithm that applies to all class $\mathcal{S}$ theories - one would like to identify and solve the $2 d$ TQFT that computes the index. The first step in this direction has been recently taken in [16]: in a limit where a single superconformal fugacity is kept (out of the original three) the $2 d$ topological theory is recognized as the zero-area limit of $q$-deformed Yang-Mills theory. In this paper we generalize this result to a two-parameter slice $(q, t)$ of the three-dimensional fugacity space, which reduces to the limit considered in [16] for $t=q$. We give a fully explicit prescription to compute this limit of the index for the most general ${ }^{4} A$-type generalized quiver of class $\mathcal{S}$.

The principle that selects this particular fugacity slice is supersymmetry enhancement, which leads to simplifications. We study systematically the limits where the index receives contributions only from states annihilated by more than one supercharge. The $(q, t)$ slice is the most general limit of this kind sensitive to the flavor fugacities associated to the punctures. We also study another interesting slice $(Q, T)$, where the index receives contribution only from "Coulomb-branch" operators, which are flavor-neutral, so the flavor dependence is lost.

Let us briefly outline the strategy of our computation. It is essential to the construction of [1] that a punctured surface $\mathcal{C}$ can be obtained, usually in more than one way, by gluing three-punctured spheres (pairs of pants) with cylinders. Different ways to decompose the same surface $\mathcal{C}$ correspond to different S-duality frames of the same SCFT. The index is a topological quantity intrinsically associated to $\mathcal{C}$ and independent of the choice of pair-of-pants decomposition. The three-punctured spheres correspond to isolated $4 d$ SCFTs, which are the elementary building blocks for all other class $\mathcal{S}$ theories. To each puncture $I$ is associated a flavor symmetry $G_{I} \subset S U(k)$ (for the $A$-type theories that we focus on). The basic gluing operation joins two maximal punctures (that is, both with $G=S U(k)$ ) and corresponds to gauging the common $S U(k)$ symmetry. It is then sufficient to give an expression for the index associated to the three-punctured spheres. Taking for illustration the SCFT associated

\footnotetext{
${ }^{3}$ We should mention that for $\mathcal{N}=1$ SCFTs obtained as IR points of an RG flow, a prescription to compute the index in terms of the UV field content and the charges of the anomaly free R-symmetry was put forward by Romelsberger [13, 17] and recently revisited with more rigor in [18]. Following the seminal work of Dolan and Osborn [19] there have been many checks and implications of this conjecture, see e.g. [20, 21, 22, 23, 24, 25].

${ }^{4}$ In particular in [16] certain overall normalization factors were determined only for theories with special types of punctures. Here we fill this gap and work in complete generality.
} 
to the sphere with three maximal punctures, its index is some function $\mathcal{I}_{q, p, t}\left(\mathbf{a}_{1}, \mathbf{a}_{2}, \mathbf{a}_{3}\right)$ of the three superconformal fugacities $(q, p, t)$ and of the $S U(k)$ flavor fugacities $\mathbf{a}_{I}=\left(a_{I}^{1}, \ldots a_{I}^{k}\right)$ at each puncture. The topological nature of the index is very constraining. It guarantees the existence of a complete basis of functions $\left\{f_{\{q, p, t\}}^{\lambda}(\mathbf{a})\right\}$, where $\lambda$ labels irreducible $S U(k)$ representations, such that $\mathcal{I}_{q, p, t}\left(\mathbf{a}_{1}, \mathbf{a}_{2}, \mathbf{a}_{3}\right)$ has the diagonal expansion

$$
\mathcal{I}_{q, p, t}\left(\mathbf{a}_{1}, \mathbf{a}_{2}, \mathbf{a}_{3}\right)=\sum_{\lambda} C(q, p, t)_{\lambda \lambda \lambda} f_{\{q, p, t\}}^{\lambda}\left(\mathbf{a}_{1}\right) f_{\{q, p, t\}}^{\lambda}\left(\mathbf{a}_{2}\right) f_{\{q, p, t\}}^{\lambda}\left(\mathbf{a}_{3}\right) .
$$

The left-hand-side is a priori unknown - except in the $S U(2)$ case, where it is the index of the free hypermultiplet theory. The idea is to focus on the explicit $S U(2)$ expression, write it in the form (1.1), and try to extrapolate the answer to general $S U(k)$. This program succeeds for the two-dimensional slice $(q, 0, t)$ in fugacity space, where the functions $f_{q, t}^{\lambda}(\mathbf{a})$ turn out to be closely related to a well-studied family of symmetric polynomials, the Macdonald polynomials, which are defined for all root systems. One is led to a compelling general conjecture that passes many tests. The extension to the three-dimensional fugacity space must be possible but is not entirely straightforward, as the basis that diagonalizes the structure constants is expected to consist of symmetric functions of an elliptic kind, which are less understood. We comment on this generalization in our conclusions.

The TQFT that computes the index turns out to be a deformation of two-dimensional Yang-Mills theory. For $t=q$ Macdonald polynomials reduce to Schur polynomials and the TQFT can be related to the zero-area limit of $2 d q$-deformed Yang-Mills theory [28, 29, 30], which can also be viewed as an analytic continuation of Chern-Simons theory on $\mathcal{C} \times S^{1}$ away from integer rank. For the more general $(q, 0, t)$ slice the TQFT appears to be closely related to the "refined" version of Chern-Simons theory recently discussed in [31].

The rest of the paper is organized as follows. In section 2 we review the definition of $\mathcal{N}=2$ superconformal index, paying special attention to the parametrization of the superconformal fugacities. In section 3 we review the TQFT structure of the index and describe the strategy of our computation. In section 4 we define interesting limits of the index characterized by enhanced supersymmetry. In section 5 we apply our strategy to the simplest limit, the fugacity slice $(0,0, t)$. In this case the functions that diagonalize the structure constants are proportional to Hall-Littlewood polynomials. We conjecture an explicit general expression and present a wide range of checks of our proposal. In particular we make contact with the results of $[32,33]$ : we show that for genus-zero quivers the HL index is equivalent to the Hilbert series of the Higgs branch and test this equivalence in several examples. In section 6 we consider the slice $(q, p, q)$ (which is in fact independent of $p$ ). This is limit of the index previously considered in [16]. The relevant symmetric functions are proportional to Schur polynomials. We generalize the results of [16] and give explicit expressions valid for arbitrary 
punctures. In section 7 we combine and generalize the results of sections 5 and 6 . We consider the fugacity slice $(q, 0, t)$ and conjecture an expression for the index associated to the general three-puncture sphere in terms of Macdonal polynomials, equation (7.9). This is our main result. In section 8 we consider an index that counts Coulomb-branch operators. Amusingly we are able to give a "physics proof" of Macdonald's constant term identities. We conclude in section 9 with a discussion of our results and speculations on a few open questions. Several appendices supplement the text with technical details and reference material.

As we were finalizing our draft we learnt about the tragic death of Francis A. Dolan. Dolan and Osborn's beautiful results on superconformal representation theory and on the index were a direct influence and inspiration for our work. This paper intersects Francis' interests in so many ways, that we knew he would be one of our most demanding readers, and aspired to be up to the standards he set. This paper is dedicated to his memory.

\section{The $\mathcal{N}=2$ superconformal index}

The superconformal index [12] encodes the information about the protected spectrum of a SCFT that can be obtained from representation theory alone. It is evaluated by a trace formula, of the schematic form

$$
\mathcal{I}\left(\mu_{i}\right)=\operatorname{Tr}(-1)^{F} e^{-\mu_{i} T_{i}} e^{-\beta \delta}, \quad \delta=2\left\{\mathcal{Q}, \mathcal{Q}^{\dagger}\right\}
$$

where $\mathcal{Q}$ is the supercharge "with respect to which" the index is calculated and $\left\{T_{i}\right\}$ a complete set of generators that commute with $\mathcal{Q}$ and with each other. The trace is over the states of the theory on $S^{d-1}$ (in the usual radial quantization). By standard arguments, states with $\delta \neq 0$ cancel pairwise, so the index counts states with $\delta=0$ (the "harmonic representatives" of the cohomology classes of $\mathcal{Q}$ ) and it is independent of $\beta$. From the index one can reconstruct the spectrum of short multiplets, up to the equivalence relations that set to zero the combinations of short multiplets that may a priori recombine into long ones [12].

For four-dimensional $\mathcal{N}=2$ SCFTs, which are non-chiral, different choices of $\mathcal{Q}$ lead to physically equivalent indices. The subalgebra of $S U(2,2 \mid 2)$ commuting with a single supercharge is $S U(1,1 \mid 2)$, which has rank three, so the $\mathcal{N}=2$ index depends on three superconformal fugacities. In addition, there will be fugacities associated with the flavor symmetries. For definiteness we choose $\mathcal{Q}=\widetilde{\mathcal{Q}}_{1-}$. See table 1 for a summary of our notations. There are three supercharges commuting with $\widetilde{\mathcal{Q}}_{1} \dot{-}$ and $\left(\widetilde{\mathcal{Q}}_{1-}\right)^{\dagger}$ :

$$
\mathcal{Q}_{1-}, \quad \mathcal{Q}_{1+}, \quad \widetilde{\mathcal{Q}}_{2+} .
$$




\begin{tabular}{|c|c|c|c|c|c|c|}
\hline $\mathcal{Q}$ & $S U(2)_{1}$ & $S U(2)_{2}$ & $S U(2)_{R}$ & $U(1)_{r}$ & $\delta$ & Commuting $\delta \mathrm{s}$ \\
\hline $\mathcal{Q}_{1-}$ & $-\frac{1}{2}$ & 0 & $\frac{1}{2}$ & $\frac{1}{2}$ & $\delta_{1-}=E-2 j_{1}-2 R-r$ & $\delta_{2+}, \quad \tilde{\delta}_{1 \dot{+}}, \quad \tilde{\delta}_{1-}$ \\
\hline $\mathcal{Q}_{1+}$ & $\frac{1}{2}$ & 0 & $\frac{1}{2}$ & $\frac{1}{2}$ & $\delta_{1+}=E+2 j_{1}-2 R-r$ & $\delta_{2-}, \quad \tilde{\delta}_{1 \dot{+}}, \quad \tilde{\delta}_{1-}$ \\
\hline $\mathcal{Q}_{2-}$ & $-\frac{1}{2}$ & 0 & $-\frac{1}{2}$ & $\frac{1}{2}$ & $\delta_{2-}=E-2 j_{1}+2 R-r$ & $\delta_{1+}, \quad \tilde{\delta}_{2 \dot{+}}, \quad \tilde{\delta}_{2-}$ \\
\hline $\mathcal{Q}_{2+}$ & $\frac{1}{2}$ & 0 & $-\frac{1}{2}$ & $\frac{1}{2}$ & $\delta_{2+}=E+2 j_{1}+2 R-r$ & $\delta_{1-}, \quad \tilde{\delta}_{2 \dot{+}}, \quad \tilde{\delta}_{2}$ \\
\hline$\widetilde{\mathcal{Q}}_{1}$ & 0 & $-\frac{1}{2}$ & $\frac{1}{2}$ & $-\frac{1}{2}$ & $\tilde{\delta}_{1-}=E-2 j_{2}-2 R+r$ & $\tilde{\delta}_{2 \dot{+}}, \quad \delta_{1+}, \quad \delta_{1-}$ \\
\hline$\widetilde{\mathcal{Q}}_{1+}$ & 0 & $\frac{1}{2}$ & $\frac{1}{2}$ & $-\frac{1}{2}$ & $\tilde{\delta}_{1 \dot{+}}=E+2 j_{2}-2 R+r$ & $\tilde{\delta}_{2-}, \quad \delta_{1+}, \quad \delta_{1-}$ \\
\hline$\widetilde{\mathcal{Q}}_{2}$ & 0 & $-\frac{1}{2}$ & $-\frac{1}{2}$ & $-\frac{1}{2}$ & $\tilde{\delta}_{2-}=E-2 j_{2}+2 R+r$ & $\tilde{\delta}_{1 \dot{+}}, \quad \delta_{2+}, \quad \delta_{2-}$ \\
\hline$\widetilde{\mathcal{Q}}_{2 \dot{+}}$ & 0 & $\frac{1}{2}$ & $-\frac{1}{2}$ & $-\frac{1}{2}$ & $\tilde{\delta}_{2 \dot{+}}=E+2 j_{2}+2 R+r$ & $\tilde{\delta}_{1-}, \quad \delta_{2+}, \quad \delta_{2-}$ \\
\hline
\end{tabular}

Table 1: For each supercharge $\mathcal{Q}$, we list its quantum numbers, the associated $\delta \equiv 2\left\{\mathcal{Q}, \mathcal{Q}^{\dagger}\right\}$, and the other $\delta$ s commuting with it. Here $I=1,2$ are $S U(2)_{R}$ indices and $\alpha= \pm, \dot{\alpha}= \pm$ Lorentz indices. $E$ is the conformal dimension, $\left(j_{1}, j_{2}\right)$ the Cartan generators of the $S U(2)_{1} \otimes S U(2)_{2}$ isometry group, and $(R, r)$, the Cartan generators of the $S U(2)_{R} \otimes U(1)_{r}$ R-symmetry group.

A useful choice is to take as a basis for the Cartan generators of the commutant subalgebra $S U(1,1 \mid 2)$ the three $\delta$ s of these supercharges. For each $\mathcal{Q}$ the associated $\delta$ is defined as

$$
\delta \equiv 2\left\{\mathcal{Q}, \mathcal{Q}^{\dagger}\right\}
$$

and it has a non-negative real spectrum. We then write the index as

$$
\mathcal{I}(\rho, \sigma, \tau)=\operatorname{Tr}(-1)^{F} \rho^{\frac{1}{2} \delta_{1-}} \sigma^{\frac{1}{2} \delta_{1+}} \tau^{\frac{1}{2} \tilde{\delta}_{2+}} e^{-\beta \tilde{\delta}_{1-}} .
$$

In table 1 we give the expressions of the $\delta$ charges in terms of the more familiar Cartan generators $\left(E, j_{1}, j_{2}, R, r\right)$ of $S U(2,2 \mid 2)$. This parametrization of the fugacities makes it easy to consider special limits with enhanced supersymmetry, which is our goal in this paper. ${ }^{5}$ Another very useful parametrization is in terms of fugacities $(p, q, t)$, related to $(\sigma, \rho, \tau)$ as

$$
p=\tau \sigma, \quad q=\tau \rho, \quad t=\tau^{2} .
$$

This is the choice that corresponds to the $(p, q)$ labels of the elliptic Gamma function [34], and also, as we shall see, to the $(t, q)$ labels of Macdonald polynomials ${ }^{6}$. In terms of these

\footnotetext{
${ }^{5}$ Although at first glance the trace formula (2.4) may seem to depend symmetrically on four equivalent $\delta \mathrm{s}$, this is not the case. The charge $\tilde{\delta}_{1}$ - is special: the associated supercharge $\widetilde{\mathcal{Q}}_{1-}$ commutes with all the four $\delta$ s, but the supercharges associated to the other three $\delta$ s do not. This is then the index "computed with respect to $\widetilde{\mathcal{Q}}_{1-}$ ", and it is independent of $\beta$, which we will usually omit.

${ }^{6}$ Note that while the fugacities $(q, p)$ have exactly the same meaning in our previous papers [14, 23, 15], the fugacity $t$ is different from the one introduced in [12] and used in $[14,23,15]$. We made this change of notations to make contact with the Macdonald literature, where $t$ has a canonical definition that one wishes to respect.
} 
fugacities, the definition of the index reads

$$
\mathcal{I}(p, q, t)=\operatorname{Tr}(-1)^{F} p^{\frac{1}{2} \delta_{1+}} q^{\frac{1}{2} \delta_{1-}} t^{R+r} e^{-\beta^{\prime} \tilde{\delta}_{1-}}
$$

In appendix $\mathrm{B}$ we review the shortening conditions of the $\mathcal{N}=2$ superconformal algebra and give the expression of the index for the various short multiplets. Given the index of a SCFT, the formulae of appendix B allow to determine its spectrum of short multiplets, up to the usual recombination ambiguities (spelled out in section 5.2 of [35]).

For a theory with a weakly-coupled description the index can be explicitly computed as a matrix integral,

$$
\mathcal{I}(V, \rho, \sigma, \tau)=\int[d U] \exp \left(\sum_{n=1}^{\infty} \frac{1}{n} \sum_{j} f^{\mathcal{R}_{j}}\left(\rho^{n}, \sigma^{n}, \tau^{n}\right) \cdot \chi_{\mathcal{R}_{j}}\left(U^{n}, V^{n}\right)\right) .
$$

Here $U$ denotes an element of the gauge group, with $[d U]$ the invariant Haar measure, and $V$ an element of the flavor group. The sum is over the different $\mathcal{N}=2$ supermultiplets appearing in the Lagrangian, with $\mathcal{R}_{j}$ the representation of the $j$-th multiplet under the flavor and gauge groups and $\chi_{\mathcal{R}_{j}}$ the corresponding character. The Haar measure has the following property

$$
\int[d U] \prod_{j=1}^{n} \chi_{\mathcal{R}_{j}}(U)=\# \text { of singlets in } \mathcal{R}_{1} \otimes \cdots \otimes \mathcal{R}_{n} .
$$

The functions $f^{(j)}$ are the "single-letter" partition functions, $f^{(j)}=f^{V}$ or $f^{(j)}=f^{\frac{1}{2} H}$ according to whether the $j$-th multiplet is an $\mathcal{N}=2$ vector or $\mathcal{N}=2 \frac{1}{2}$-hypermultiplet. The "single letters" of an $\mathcal{N}=2$ gauge theory contributing to the index obey $\tilde{\delta}_{1-}=E-2 j_{2}-2 R+r=0$ and are enumerated in table 2 . The first block of table 2 shows the contributing letters from the $\mathcal{N}=2$ vector multiplet, including the equations of motion constraint. The second block shows the contributions from the half-hypermultiplet (or $\mathcal{N}=1$ chiral multiplet). The last line shows the spacetime derivatives contributing to the index. Since each field can be hit by an arbitrary number of derivatives, the derivatives give a multiplicative contribution to the single-letter partition functions of the form

$$
\sum_{m=0}^{\infty} \sum_{n=0}^{\infty}(\rho \tau)^{m}(\sigma \tau)^{n}=\frac{1}{(1-\rho \tau)(1-\sigma \tau)} .
$$

The single-letter partition functions of the $\mathcal{N}=2$ vector and $\mathcal{N}=1$ chiral multiplets are thus given by

$$
\begin{aligned}
f^{V} & =-\frac{\sigma \tau}{1-\sigma \tau}-\frac{\rho \tau}{1-\rho \tau}+\frac{\sigma \rho-\tau^{2}}{(1-\rho \tau)(1-\sigma \tau)} \\
& =-\frac{p}{1-p}-\frac{q}{1-q}+\frac{p q / t-t}{(1-q)(1-p)}, \\
f^{\frac{1}{2} H} & =\frac{\tau}{(1-\rho \tau)(1-\sigma \tau)}(1-\rho \sigma)=\frac{\sqrt{t}-p q / \sqrt{t}}{(1-q)(1-p)} .
\end{aligned}
$$




\begin{tabular}{|c|r|r|r|r|r|c|c|}
\hline Letters & $E$ & $j_{1}$ & $j_{2}$ & $R$ & $r$ & $\mathcal{I}(\sigma, \rho, \tau)$ & $\mathcal{I}(p, q, t)$ \\
\hline \hline$\phi$ & 1 & 0 & 0 & 0 & -1 & $\sigma \rho$ & $p q / t$ \\
\hline$\lambda_{1 \pm}$ & $\frac{3}{2}$ & $\pm \frac{1}{2}$ & 0 & $\frac{1}{2}$ & $-\frac{1}{2}$ & $-\sigma \tau,-\rho \tau$ & $-p,-q$ \\
\hline $\bar{\lambda}_{1 \dot{+}}$ & $\frac{3}{2}$ & 0 & $\frac{1}{2}$ & $\frac{1}{2}$ & $\frac{1}{2}$ & $-\tau^{2}$ & $-t$ \\
\hline $\bar{F}_{\dot{+}+}$ & 2 & 0 & 1 & 0 & 0 & $\sigma \rho \tau^{2}$ & $p q$ \\
\hline$\partial_{-\dot{+}} \lambda_{1+}+\partial_{+\dot{+}} \lambda_{1-}=0$ & $\frac{5}{2}$ & 0 & $\frac{1}{2}$ & $\frac{1}{2}$ & $-\frac{1}{2}$ & $\sigma \rho \tau^{2}$ & $p q$ \\
\hline \hline$q$ & 1 & 0 & 0 & $\frac{1}{2}$ & 0 & $\tau$ & $\sqrt{t}$ \\
\hline $\bar{\psi}_{\dot{+}}$ & $\frac{3}{2}$ & 0 & $\frac{1}{2}$ & 0 & $-\frac{1}{2}$ & $-\sigma \rho \tau$ & $-p q / \sqrt{t}$ \\
\hline \hline$\partial_{ \pm \dot{+}}$ & 1 & $\pm \frac{1}{2}$ & $\frac{1}{2}$ & 0 & 0 & $\sigma \tau, \rho \tau$ & $p, q$ \\
\hline
\end{tabular}

Table 2: Contributions to the index from "single letters". We denote by $\left(\phi, \bar{\phi}, \lambda_{I, \alpha}, \bar{\lambda}_{I \dot{\alpha}}, F_{\alpha \beta}, \bar{F}_{\dot{\alpha} \dot{\beta}}\right)$ the components of the adjoint $\mathcal{N}=2$ vector multiplet, by $\left(q, \bar{q}, \psi_{\alpha}, \bar{\psi}_{\dot{\alpha}}\right)$ the components of the $\mathcal{N}=1$ chiral multiplet, and by $\partial_{\alpha \dot{\alpha}}$ the spacetime derivatives.

For general values of the three fugacities the explicit expression for the index of a Lagrangian theory is most elegantly expressed [19] in terms of the elliptic Gamma functions (see [34] for a nice review of these special functions). In this paper however we consider reduced forms of the index and do not utilize the power of these special functions. We comment on the relation to elliptic functions in the concluding section 9.

\section{TQFT structure of the index}

Four-dimensional superconformal field theories of $\mathcal{S}[1,2]$ arise from partially-twisted compactification of the six-dimensional $(2,0)$ theory on a punctured Riemann surface $\mathcal{C}$. The complex-structure moduli of $\mathcal{C}$ are identified with the exactly marginal couplings of the $4 d$ SCFT, while the punctures are associated to flavor symmetries.

Any punctured surface can be obtained, usually in more than one way, by gluing threepunctured spheres (pairs of pants) with cylinders. The three-punctured spheres are then the elementary building blocks. They correspond to isolated $4 d$ SCFTs with flavor symmetry $G_{1} \otimes G_{2} \otimes G_{3}$, where each factor $G_{I}$ is associated to one of the three punctures. ${ }^{7}$ The cylinders correspond to $\mathcal{N}=2$ vector multiplets, and the gluing operation amounts to gauging

\footnotetext{
${ }^{7}$ In this paper we focus on class $\mathcal{S}$ theories that descend from the $(2,0)$ theory of type $A_{k-1}$. Then the punctures are classified by the possible embeddings of $S U(2)$ into $S U(k)$ and $G_{I} \subset S U(k)$ is the commutant of the chosen embedding.
} 
a common $S U(k)$ symmetry of two punctures. The gluing parameter is interpreted as the complexified gauge coupling, with zero coupling corresponding to an infinitely long cylinder a degeneration limit of the surface. Different pairs-of-pants decompositions of the same surface $\mathcal{C}$ correspond to different descriptions of the same SCFT, related by generalized S-dualities.

Since the index is independent of the moduli, and is invariant under S-dualities, it is naturally viewed as a correlator in a $2 d$ topological QFT living on $\mathcal{C}$ [14]. Let us review how this works. We parametrize the index of a three-punctured sphere as $\mathcal{I}\left(\mathbf{a}_{1}, \mathbf{a}_{2}, \mathbf{a}_{3}\right)$, where $\mathbf{a}_{I}$ are fugacities dual to the Cartan subgroup of $G_{I}$ : except in special cases these are a priori unknown functions. On the other hand we can easily write down the "propagator" associated to a cylinder,

$$
\eta(\mathbf{a}, \mathbf{b})=\Delta(\mathbf{a}) \mathcal{I}^{V}(\mathbf{a}) \delta\left(\mathbf{a}, \mathbf{b}^{-1}\right)
$$

where $\Delta(\mathbf{a})$ is the Haar measure and $\mathcal{I}^{V}(\mathbf{a})$ the index of a vector multiplet, which is known explicitly. The index of a generic theory of class $\mathcal{S}$ can be written in terms of the index of these elementary constituents. As the simplest example, gluing two three-punctured spheres with one cylinder one obtains the index of a four-punctured sphere,

$$
\begin{aligned}
\mathcal{I}\left(\mathbf{a}_{1}, \mathbf{a}_{2}, \mathbf{a}_{3}, \mathbf{a}_{4}\right) & =\oint[d \mathbf{a}] \oint[d \mathbf{b}] \mathcal{I}\left(\mathbf{a}_{1}, \mathbf{a}_{2}, \mathbf{a}\right) \eta(\mathbf{a}, \mathbf{b}) \mathcal{I}\left(\mathbf{b}, \mathbf{a}_{3}, \mathbf{a}_{4}\right) \\
& =\oint[d \mathbf{a}] \Delta(\mathbf{a}) \mathcal{I}\left(\mathbf{a}_{1}, \mathbf{a}_{2}, \mathbf{a}\right) \mathcal{I}^{V}(\mathbf{a}) \mathcal{I}\left(\mathbf{a}^{-1}, \mathbf{a}_{3}, \mathbf{a}_{4}\right)
\end{aligned}
$$

where we have introduced the notation

$$
\oint[d \mathbf{a}] \equiv \oint \prod_{i=1}^{k-1} \frac{d a_{i}}{2 \pi i a_{i}}
$$

If we expand the index in a convenient basis of functions $\left\{f^{\alpha}(\mathbf{a})\right\}$, labeled by $S U(k)$ representations $\{\alpha\},{ }^{8}$ we can associate to each three-punctured sphere "structure constants" $C_{\alpha \beta \gamma}$ and to each propagator a metric $\eta^{\alpha \beta}$,

$$
\begin{aligned}
\mathcal{I}(\mathbf{a}, \mathbf{b}, \mathbf{c}) & =\sum_{\alpha, \beta, \gamma} C_{\alpha \beta \gamma} f^{\alpha}(\mathbf{a}) f^{\beta}(\mathbf{b}) f^{\gamma}(\mathbf{c}) \\
\eta^{\alpha \beta} & =\oint[d \mathbf{a}] \oint[d \mathbf{b}] \eta(\mathbf{a}, \mathbf{b}) f^{\alpha}(\mathbf{a}) f^{\beta}(\mathbf{b}) .
\end{aligned}
$$

\footnotetext{
${ }^{8}$ For theories of type $A,\left\{f^{\alpha}(\mathbf{a})\right\}$ are symmetric functions of their arguments, which are fugacities dual to the Cartan generators of $S U(k)$. More generally, for theories of type $D$ and $E,\left\{f^{\alpha}(\mathbf{a})\right\}$ are invariant under the appropriate Weyl group.
} 
Invariance of the index under the different ways to decompose the surface is tantamount of saying that $C_{\alpha \beta \gamma}$ and $\eta^{\alpha \beta}$ define a two-dimensional topological QFT. ${ }^{9}$ The crucial property is associativity,

$$
C_{\alpha \beta \gamma} C_{\delta \epsilon}^{\gamma}=C_{\alpha \delta \gamma} C_{\beta \epsilon}^{\gamma},
$$

where indices are raised with the metric $\eta^{\alpha \beta}$ and lowered with the inverse metric $\eta_{\alpha \beta}$.

It is very natural to choose the complete set of functions $\left\{f^{\alpha}(\mathbf{a})\right\}$ to be orthonormal under the measure that appears in the propagator,

$$
\oint[d \mathbf{a}] \Delta(\mathbf{a}) \mathcal{I}^{V}(\mathbf{a}) f^{\alpha}(\mathbf{a}) f^{\beta}\left(\mathbf{a}^{-1}\right)=\delta^{\alpha \beta} .
$$

Then the metric $\eta^{\alpha \beta}$ is trivial,

$$
\eta^{\alpha \beta}=\delta^{\alpha \beta}
$$

Condition (3.7) still leaves considerable freedom, as it is obeyed by infinitely many bases of functions related by orthogonal transformations. The real simplification arises if we can find an explicit basis $\left\{f^{\alpha}(\mathbf{a})\right\}$, such that the structure constants are diagonal,

$$
C_{\alpha \beta \gamma} \neq 0 \quad \rightarrow \quad \alpha=\beta=\gamma
$$

Associativity (3.6) is then automatic. For structure constants satisfying (3.6) one can always find a basis in which they are diagonal: we give a detailed example of such a diagonalization procedure in appendix A for the simplest limit of the index. The challenge is to describe the basis in concrete form.

In general the measure appearing in the propagator is complicated and no explicit set of orthonormal functions is available. We find it very useful to consider an ansatz

$$
f^{\alpha}(\mathbf{a})=\mathcal{K}(\mathbf{a}) P^{\alpha}(\mathbf{a})
$$

for some function $\mathcal{K}(\mathbf{a})$. Clearly, from (3.7), the functions $\left\{P^{\alpha}(\mathbf{a})\right\}$ are orthornormal under the new measure $\hat{\Delta}(\mathbf{a})$,

$$
\oint[d \mathbf{a}] \hat{\Delta}(\mathbf{a}) P^{\alpha}(\mathbf{a}) P^{\beta}\left(\mathbf{a}^{-1}\right)=\delta^{\alpha \beta}, \quad \hat{\Delta}(\mathbf{a}) \equiv \mathcal{I}^{V}(\mathbf{a}) \mathcal{K}(\mathbf{a})^{2} \Delta(\mathbf{a})
$$

\footnotetext{
${ }^{9}$ We are using this term somewhat loosely. As axiomatized by Atiyah, a TQFT is understood to have a finite-dimensional state-space, while in our case the state-space will be infinite-dimensional. The bestunderstood example of a $2 d$ topological theory with an infinite-dimensional state-space is the zero-area limit of $2 d$ Yang-Mills theory [36, 37] (see e.g. [38] for a comprehensive review). Happily, the $2 d$ topological theory associated to the index turns out to be closely related to $2 d$ Yang-Mills.
} 
(Recall that $\Delta(\mathbf{a})$ always denotes the Haar measure). The name of the game is to find a clever choice of $\mathcal{K}(\mathbf{a})$, for which $\hat{\Delta}(\mathbf{a})$ is a simple known measure and the orthonormal basis $\left\{P^{\alpha}(\mathbf{a})\right\}$ an explicit set of functions such that (3.9) holds.

Once the diagonal basis $\left\{f^{\alpha}(\mathbf{a})\right\}$ and the structure constant $C_{\alpha \alpha \alpha}$ are known, one can easily calculate the index of the SCFT associated to the genus $\mathfrak{g}$ surface with $s$ punctures. Such a surface can be built by gluing $2 \mathfrak{g}-2+s$ three-punctured spheres, so we have ${ }^{10}$

$$
\mathcal{I}_{\mathfrak{g}, s}\left(\mathbf{a}_{1}, \mathbf{a}_{2}, \ldots, \mathbf{a}_{s}\right)=\sum_{\alpha}\left(C_{\alpha \alpha \alpha}\right)^{2 \mathfrak{g}-2+s} \prod_{I=1}^{s} f^{\alpha}\left(\mathbf{a}_{I}\right) .
$$

In the rest of the paper we implement the following strategy. We start by considering the generalized $S U(2)$ quivers. Since they have a Lagrangian description, closed form expressions for the index (as matrix integrals) are readily available. We then look for a basis of functions $\left\{f^{\alpha}(\mathbf{a})\right\}$ that diagonalizes the structure constants. Fortunately, for each special limit of the index that we consider, the diagonal basis is of the form (3.10), with $\left\{P^{\alpha}(\mathbf{a})\right\}$ well-known symmetric polynomials: Hall-Littlewood, Schur or Macdonald polynomials. (The first two are in fact special cases of Macdonald polynomials). Since these polynomials are defined for arbitrary rank, we can extrapolate from the $S U(2)$ case and formulate compelling conjectures for the index of all generalized quivers of type $A$. (This approach readily generalizes to all $A D E$ theories, but in this paper we focus on the $A$ series). Finally we check our conjectures against expected symmetry enhancements and S-dualities.

\section{Limits of the index with additional supersymmetry}

We now consider several limits of the superconformal index, such that the states contributing to it are annihilated by more than one supercharge. Recall that before taking any limit the index receives contributions only from states with

$$
\tilde{\delta}_{1-}=E-2 j_{2}-2 R+r=0
$$

which are annihilated by $\widetilde{\mathcal{Q}}_{1-}$. We tend to refer to the different limits of the index by the type of symmetric polynomials relevant for their evaluation. In appendix B we discuss which short multiplets of the superconformal algebra are counted by the index in each of these limits.

\footnotetext{
${ }^{10}$ Here for simplicity we are considering the case where all external punctures are "maximal", i.e. they have flavor symmetry $S U(k)$. The prescription for punctures with reduced symmetry is discussed in detail in sections 5,6 and 7 .
} 


\section{Macdonald index}

We first consider the limit ${ }^{11}$

$$
\sigma \rightarrow 0, \quad \rho, \tau \text { fixed }
$$

(which is the same as $p \rightarrow 0$ with $q$ and $t$ fixed). The limit is well-defined since the power of $\sigma$ in the trace formula (2.4) is given by $\frac{1}{2} \delta_{1+} \geq 0$. The index is given by

$$
\begin{aligned}
\mathcal{I}_{M} & =\operatorname{Tr}_{M}(-1)^{F} \rho^{\frac{1}{2}\left(E-2 j_{1}-2 R-r\right)} \tau^{\frac{1}{2}\left(E+2 R+2 j_{2}+r\right)} \\
& =\operatorname{Tr}_{M}(-1)^{F} q^{\frac{1}{2}\left(E-2 j_{1}-2 R-r\right)} t^{R+r},
\end{aligned}
$$

where $\operatorname{Tr}_{M}$ denotes the trace restricted to states with $\delta_{1+}=E+2 j_{1}-2 R-r=0$. Such states are annihilated by $\mathcal{Q}_{1+}$. All in all $\mathcal{I}_{M}$ is a $\frac{1}{4}$-BPS object receiving contributions only from states annihilated by two supercharges, one chiral $\left(\mathcal{Q}_{1+}\right)$ and one anti-chiral $\left(\widetilde{\mathcal{Q}}_{1-}\right)$. The single letter partition functions of the half-hypermultiplet and the vector simplify to

$$
f^{\frac{1}{2} H}=\frac{\tau}{1-\rho \tau}=\frac{\sqrt{t}}{1-q}, \quad f^{V}=\frac{-\tau^{2}-\rho \tau}{1-\rho \tau}=\frac{-t-q}{1-q} .
$$

\section{Hall-Littlewood index}

We further specialize the index by sending $\rho \rightarrow 0$, so we are taking the limit

$$
\sigma \rightarrow 0, \quad \rho \rightarrow 0, \quad \tau \text { fixed }
$$

(equivalently, $q, p \rightarrow 0$ with $t$ fixed), which is well-defined thanks to $\delta_{1 \pm} \geq 0$. The index is given by

$$
\mathcal{I}_{H L}=\operatorname{Tr}_{H L}(-1)^{F} \tau^{\frac{1}{2}\left(E+2 R+2 j_{2}+r\right)}=\operatorname{Tr}_{H L}(-1)^{F} \tau^{2(E-R)},
$$

where $\operatorname{Tr}_{H L}$ denotes the trace restricted to states with $\delta_{1 \pm}=E \pm 2 j_{1}-2 R-r=0$. All in all, taking (4.1) into account, the states contributing to the index obey

$$
j_{1}=0, \quad j_{2}=r, \quad E=2 R+r,
$$

and are annihilated by three supercharges: $\mathcal{Q}_{1+}, \mathcal{Q}_{1-}$ and $\widetilde{\mathcal{Q}}_{1-}$.

Let us consider the Hall-Littlewood (HL) index for a theory with a Lagrangian description. From table 2, we see that it gets contributions only from the scalar $q$ of the hypermultiplet and from the fermion $\bar{\lambda}_{1 \dot{+}}$ of the vector multiplet. The single letter partition function of the half-hypermultiplet and the vector multiplet is then

$$
f^{\frac{1}{2} H}=\tau, \quad f^{V}=-\tau^{2} .
$$

\footnotetext{
${ }^{11}$ An equivalent limit can be obtained by sending $\rho$ to zero.
} 
Remarkably, for generalized quivers with a sphere topology the computation of the HL index is equivalent to the computation of the partition function over the Higgs branch discussed in $\left[32,33\right.$ ] (the Hilbert series of the Higgs branch). ${ }^{12}$ This can be shown as follows. To compute the partition function of $[32,33]$ for the Higgs branch of an $\mathcal{N}=2$ gauge theory one counts all the possible gauge invariant operators built from the scalar components of the hypermultiplets taking into account the F-term superpotential constraints. In an $\mathcal{N}=2$ gauge theory with $M S U(2)$ gauge factors the superpotential takes the form

$$
W=\sum_{i=1}^{M} \sum_{\alpha \in\{i\}} Q_{a_{i} a_{k} a_{l}}^{(\alpha)} \Phi_{b_{i}}^{a_{i}} Q^{(\alpha) b_{i} a_{k} a_{l}},
$$

where the summation over $i$ is over the gauged groups. The set $\{i\}$ is the set of (at most two) trifundamental hypermultiplets transforming non-trivially under gauge group $i$. The F-term constraints then read

$$
Q_{a_{i} a_{k} a_{l}}^{\left(\alpha_{1}\right)} Q^{\left(\alpha_{1}\right) b_{i} a_{k} a_{l}}+Q_{a_{i} a_{m} a_{n}}^{\left(\alpha_{2}\right)} Q^{\left(\alpha_{2}\right) b_{i} a_{m} a_{n}}=0 .
$$

If the quiver diagram does not have loops, i.e. the corresponding Riemann surface has a topology of a sphere, this is a set of $M$ independent constraints. It then follows that the computation of this partition function is the same as the computation of the index. Indeed, one associates a fugacity $\tau$ for each scalar component of $Q$. The constraint (4.10) is quadratic in $Q$ and is in the adjoint representation of the gauge group. It is implemented by multiplying the unconstrained partition function with the following factor [32, 33],

$$
\exp \left[-\sum_{n=1}^{\infty} \frac{1}{n} \tau^{2 n}\left(a_{i}^{2 n}+a_{i}^{-2 n}+1\right)\right]=\left(1-\tau^{2}\right)\left(1-\tau^{2} a_{i}^{2}\right)\left(1-\tau^{2} a_{i}^{-2}\right) .
$$

This factor is the same as the index of the letter $\bar{\lambda}_{1 \dot{+}}$. Thus, one can think of the letter $\bar{\lambda}_{1 \dot{+}}$ in the calculation of the index as playing the same role as the superpotential constraint in the calculation of the Higgs partition function! This logic can be extended to higher-rank theories, where not all the building blocks have Lagrangian description, but the Higgs branch can still be described in terms of operators obeying certain constraints. This concludes the argument that the HL index is the same as the Higgs partition function for theories with sphere topology. Our derivation also makes it clear that this correspondence fails for highergenus theories.

In [32] non-trivial very explicit expressions for the Higgs branch partition function of the SCFTs with exceptional flavor symmetry groups [27, 41] were conjectured. We will see that they are exactly reproduced by the HL index.

\footnotetext{
${ }^{12}$ A relation of a similar limit of the $\mathcal{N}=1$ index with the counting problems discussed in [39, 40] was mentioned in [21]. We thank V. Spiridonov for bringing this reference to our attention.
} 


\section{Schur index}

The Schur index is defined by specializing the fugacities to $\rho=\tau$ with $\sigma$ arbitrary (equivalently $q=t$ with $p$ arbitrary). It reads

$$
\mathcal{I}_{S}=\operatorname{Tr}(-1)^{F} \sigma^{\frac{1}{2}\left(E+2 j_{1}-2 R-r\right)} \rho^{E-j_{1}+j_{2}} e^{-\beta\left(E-2 j_{2}-2 R+r\right)} .
$$

By construction, all charges in the trace formula commute with the supercharge $\widetilde{\mathcal{Q}}_{1}$ - "with respect to which" the index is evaluated. From table 1, we observe that the charges in (4.12) also commute with $\mathcal{Q}_{1+}$. Thus the index receives contributions from states with $\delta_{1+}=\tilde{\delta}_{1-}=0$ (the intersection of the cohomologies of $\mathcal{Q}_{1+}$ and of $\widetilde{\mathcal{Q}}_{1 \dot{-}}$ ) and it is independent of both $\sigma$ and $\beta$. We can then write

$$
\mathcal{I}_{S}=\operatorname{Tr}(-1)^{F} \rho^{2(E-R)}=\operatorname{Tr}(-1)^{F} q^{E-R}
$$

The Schur index can also be obtained as a special case of the Macdonald index by setting $\rho=\tau$ (equivalently $q=t$ ); we have just seen that for $\rho=\tau$ the index becomes independent of $\sigma$ so the limit $\sigma \rightarrow 0$ that we take to obtain the Macdonald index is immaterial.

The single letter partition functions of the half-hypermultiplets and the vector multiplet are given by

$$
f^{\frac{1}{2} H}=\frac{\rho}{1-\rho^{2}}=\frac{\sqrt{q}}{1-q}, \quad f^{V}=\frac{-2 \rho^{2}}{1-\rho^{2}}=\frac{-2 q}{1-q} .
$$

The Schur index is the same as the index studied in [16], where we referred to it as the reduced index.

\section{Coulomb-branch index}

Finally we consider the limit

$$
\tau \rightarrow 0, \quad \rho, \sigma \text { fixed }
$$

which is well-defined thanks to $\tilde{\delta}_{2 \dot{+}} \geq 0$. The trace formula becomes

$$
\mathcal{I}_{C}=\operatorname{Tr}_{C}(-1)^{F} \sigma^{\frac{1}{2}\left(E+2 j_{1}-2 R-r\right)} \rho^{\frac{1}{2}\left(E-2 j_{1}-2 R-r\right)} e^{-\beta\left(E-2 j_{2}-2 R+r\right)},
$$

where $\operatorname{Tr}_{C}$ denotes the trace over the states with $\tilde{\delta}_{2 \dot{+}}=E+2 j_{2}+2 R+r=0$, which are

annihilated by $\widetilde{\mathcal{Q}}_{2 \dot{+}}$. All in all, the index gets contributions from states annihilated by two antichiral supercharges, $\widetilde{\mathcal{Q}}_{1-}$ and $\widetilde{\mathcal{Q}}_{2 \dot{+}}$.

In this limit the single-letter partition function of the half-hypermultiplet and the vector multiplet are

$$
f^{\frac{1}{2} H}=0, \quad f^{V}=\sigma \rho \equiv T .
$$


From the viewpoint of the the single-letter partition functions one can take an interesting less restrictive limit,

$$
\tau, \sigma \rightarrow 0, \quad \rho \rightarrow \infty \quad \text { with } \quad Q \equiv \tau \rho \text { and } T \equiv \sigma \rho \text { fixed. }
$$

In this limit we have

$$
f^{\frac{1}{2} H}=0, \quad f^{V}=\frac{T-Q}{1-Q} .
$$

We recover (4.17) for $Q \rightarrow 0$. In terms of the new fugacities $Q$ and $T$ the index reads

$$
\mathcal{I}_{C M}=\operatorname{Tr}_{C M}(-1)^{F} T^{\frac{1}{2}\left(E+2 j_{1}-2 R-r\right)} Q^{\frac{1}{2}\left(E+2 j_{2}+2 R+r\right)},
$$

where $\operatorname{Tr}_{C M}$ denotes the trace restricted to states satisfying $E+2 j_{1}+r=0$. This index is well-defined for Lagrangian theories and for theories related to them by dualities.

We now describe the explicit evaluation of these special limits of the index for the SCFTs of class $\mathcal{S}$.

\section{Hall-Littlewood index}

We begin with the Hall-Littlewood index,

$$
\mathcal{I}_{H L}(\tau)=\operatorname{Tr}_{H L}(-1)^{F} \tau^{2 E-2 R},
$$

where $\operatorname{Tr}_{H L}$ denotes the trace restricted to states with $j_{1}=0$ and $E-2 R-r=0$. This is the limit that leads to the greatest simplifications.

\section{$5.1 S U(2)$ quivers}

Let us start from the $S U(2)$ generalized quivers, for which the basic building blocks are known explicitly. There is only one type of non-trivial puncture, the maximal puncture with $S U(2)$ flavor symmetry. The SCFT corresponding the three-punctured sphere, denoted by $T_{2}$ in [1], is the theory of free hypermultiplets in the trifundamental representation of $S U(2)$. Its index is immediately evaluated,

$$
\mathcal{I}(a, b, c)=P E\left[\tau \chi_{1}(a) \chi_{1}(b) \chi_{1}(c)\right]_{a, b, c, \tau}=\frac{1}{\prod_{s_{a}, s_{b}, s_{c}= \pm 1}\left(1-\tau a^{s_{a}} b^{s_{b}} c^{s_{c}}\right)},
$$

where the fugacities $a, b$, and $c$ label the Cartans of the three $S U(2)$ flavor groups. The plethystic exponent $P E$ is defined as

$$
P E\left[f\left(x_{i}\right)\right]_{x_{i}} \equiv \exp \left(\sum_{n=1}^{\infty} \frac{1}{n} f\left(x_{i}^{n}\right)\right) .
$$


We will often omit the subscript $x_{i}$ in the expressions for $P E[\ldots] \cdot \chi_{1}(a)$ is the character of fundamental representation of $S U(2)$. More generally the $S U(2)$ Schur polynomials $\chi_{\lambda}$ are given by

$$
\chi_{\lambda}(a)=\frac{a^{-1-\lambda}-a^{1+\lambda}}{a^{-1}-a} .
$$

The propagator $\eta(a, b)$ is also easily evaluated:

$$
\eta(a, b)=\Delta(a) \mathcal{I}^{V}(a) \delta\left(a, b^{-1}\right)
$$

where $\mathcal{I}^{V}(a)$ is the index of the vector multiplet,

$$
\mathcal{I}^{V}(a)=P E\left[-\tau^{2} \chi_{2}(a)\right]_{a, \tau}=\left(1-\tau^{2}\right)\left(1-\tau^{2} a^{2}\right)\left(1-\tau^{2} a^{-2}\right),
$$

and $\Delta(a)$ the $S U(2)$ Haar measure,

$$
\Delta(a)=\frac{1}{2}\left(1-a^{2}\right)\left(1-\frac{1}{a^{2}}\right) .
$$

Following the strategy outlined in section 3, we look for a complete set of functions $\left\{f^{\lambda}(a)\right\}$ orthonormal under the propagator measure such that the structure constants are diagonal,

$$
\mathcal{I}(a, b, c)=\sum_{\lambda=0}^{\infty} C_{\lambda \lambda \lambda} f^{\lambda}(a) f^{\lambda}(b) f^{\lambda}(c) .
$$

We describe this calculation in appendix A. We find the remarkable result

$$
\begin{aligned}
f^{\lambda}(a) & =\mathcal{K}(a) P_{H L}^{\lambda}\left(a, a^{-1} \mid \tau\right), \\
C_{\lambda \lambda \lambda} & =\frac{\sqrt{1-\tau^{2}}\left(1+\tau^{2}\right)}{P_{H L}^{\lambda}\left(\tau, \tau^{-1} \mid \tau\right)} .
\end{aligned}
$$

Here $P_{H L}^{\lambda}$ are the $S U(2)$ Hall-Littlewood polynomials,

$$
P_{H L}^{\lambda}\left(a, a^{-1} \mid \tau\right)=\chi_{\lambda}(a)-\tau^{2} \chi_{\lambda-2}(a) \quad \text { for } \lambda \geq 1, \quad P_{H L}^{\lambda=0}\left(a, a^{-1} \mid \tau\right)=\sqrt{1+t^{2}}
$$

which are orthonormal under the measure

$$
\hat{\Delta}(a)=\Delta_{H L}(a)=\frac{1}{2} \frac{\left(1-a^{2}\right)\left(1-a^{-2}\right)}{\left(1-\tau^{2} a^{2}\right)\left(1-\tau^{2} a^{-2}\right)} .
$$

The requirement that $\left\{f^{\lambda}(a)\right\}$ be orthonormal under the propagator measure $\Delta(a) \mathcal{I}^{V}(a)$ fixes the prefactor $\mathcal{K}(a)$,

$$
\mathcal{K}(a)=\left(\frac{\Delta_{H L}(a)}{\Delta(a) \mathcal{I}^{V}(a)}\right)^{\frac{1}{2}}=\frac{1}{\sqrt{1-\tau^{2}}} \frac{1}{\left(1-\tau^{2} a^{2}\right)\left(1-\tau^{2} a^{-2}\right)}
$$


We can now immediately write down an explicit formula for the index of any generalized $S U(2)$ quiver associated to a genus $\mathfrak{g}$ Riemann surface with $s$ punctures. From (3.12),

$$
\begin{aligned}
\mathcal{I}_{\mathfrak{g}, s}\left(a_{1}, a_{2}, \ldots, a_{s}\right)= & \left(1-\tau^{2}\right)^{\mathfrak{g}-1}\left(1+\tau^{2}\right)^{2 \mathfrak{g}-2+s} . \\
& \sum_{\lambda=0}^{\infty} \frac{1}{\left[P_{H L}^{\lambda}\left(\tau, \tau^{-1} \mid \tau\right)\right]^{2 \mathfrak{g}-2+s}} \prod_{I=1}^{s} \frac{P_{H L}^{\lambda}\left(a_{I}, a_{I}^{-1} \mid \tau\right)}{\left(1-\tau^{2} a_{I}^{2}\right)\left(1-\tau^{2} a_{I}^{-2}\right)} .
\end{aligned}
$$

In particular for genus $\mathfrak{g}$ with no punctures the sum over the $S U(2)$ irreducible representations in (5.14) can be explicitly performed and one gets

$$
\mathcal{I}_{\mathfrak{g}}^{(2)}=\frac{\left(1-\tau^{2}\right)^{\mathfrak{g}-1}\left(\tau^{2 \mathfrak{g}-2}+\left(1+\tau^{2}\right)^{\mathfrak{g}-1}\left(1-\tau^{2 \mathfrak{g}-2}\right)\right)}{1-\tau^{2 \mathfrak{g}-2}} .
$$

We observe that setting a flavor fugacity $a=\tau$ we "close" the corresponding puncture. For example we can go from the three-punctured sphere to the two-punctured sphere (=cylinder),

$$
\mathcal{I}\left(a_{1}, a_{2}, \tau\right) \sim \sum_{\lambda} P_{H L}^{\lambda}\left(a_{1}, a_{1}^{-1} \mid \tau\right) P_{H L}^{\lambda}\left(a_{2}, a_{2}^{-1} \mid \tau\right)=\eta\left(a_{1}, a_{2}\right)
$$

(There is an overall divergent proportionality factor). This procedure of (partially) closing punctures by trading (some of) the flavor fugacities with $\tau$ plays an important role, as it will allow us to construct the index for theories with arbitrary types of punctures. For $S U(k)$ theories the punctures are classified by the different embeddings of $S U(2)$ inside $S U(k)[1,2]$, which are conveniently labelled by auxiliary Young diagrams with $k$ boxes. For $S U(2)$ we get only two possibilities: (i) a row with two boxes corresponding to the "maximal" puncture with $S U(2)$ flavor symmetry, (ii) a column with two boxes corresponding to the absence of a puncture. For higher-rank theories the space of possibilities will be more interesting.

\subsection{Higher rank: preliminaries}

For higher-rank quivers the situation is more complicated since the basic building blocks are given by strongly-interacting SCFTs for which direct computations are not possible. However, the expressions that we obtained for the index of the $S U(2)$ quivers can be naturally extrapolated to higher rank. The basic conjecture is that the set of functions $\left\{f^{\alpha}(\mathbf{a})\right\}$ that diagonalize the structure constants are related to Hall-Littlewood polynomials for higher-rank as well.

The Hall-Littlewood (HL) polynomials associated to $U(k)$ are a set of orthogonal polynomials labeled by Young diagrams with at most $k$ rows, $\lambda=\left(\lambda_{1}, \ldots, \lambda_{k}\right), \lambda_{j} \geq \lambda_{j+1}$. They 
are given by [42]

$$
P_{H L}^{\lambda}\left(x_{1}, \ldots, x_{k} \mid \tau\right)=\mathcal{N}_{\lambda}(\tau) \sum_{\sigma \in S_{k}} x_{\sigma(1)}^{\lambda_{1}} \ldots x_{\sigma(k)}^{\lambda_{k}} \prod_{i<j} \frac{x_{\sigma(i)}-\tau^{2} x_{\sigma(j)}}{x_{\sigma(i)}-x_{\sigma(j)}},
$$

and they are orthonormal under the measure

$$
\Delta_{H L}=\frac{1}{k !} \prod_{i \neq j} \frac{1-x_{i} / x_{j}}{1-\tau^{2} x_{i} / x_{j}}
$$

The normalization $\mathcal{N}_{\lambda}(t)$ is given by

$$
\mathcal{N}_{\lambda_{1}, \ldots \lambda_{k}}^{-2}(\tau)=\prod_{i=0}^{\infty} \prod_{j=1}^{m(i)}\left(\frac{1-\tau^{2 j}}{1-\tau^{2}}\right)
$$

where $m(i)$ is the number of rows in the Young diagram $\lambda=\left(\lambda_{1}, \ldots, \lambda_{k}\right)$ of length $i$. For $S U(k)$ groups we take Young diagrams with $\lambda_{k}=0$ and the product of $x_{k}$ in (5.17) is constrained as $\prod_{i=1}^{k} x_{k}=1$.

Let us also quote from the outset the expression for the $S U(k)$ propagator,

$$
\eta\left(\mathbf{a}, \mathbf{b}^{-1}\right)=\Delta(\mathbf{a}) \mathcal{I}^{V}(\mathbf{a}) \delta\left(\mathbf{a}, \mathbf{b}^{-1}\right)
$$

where $\Delta(\mathbf{a})$ is the $S U(k)$ Haar measure,

$$
\Delta(\mathbf{a})=\frac{1}{k !} \prod_{i \neq j}\left(1-\frac{a_{i}}{a_{j}}\right), \quad \prod_{i}^{k} a_{i}=1
$$

and $\mathcal{I}^{V}(\mathbf{a})$ the vector multiplet index,

$$
\mathcal{I}^{V}=\frac{1}{1-\tau^{2}} \prod_{j, i=1}^{k}\left(1-\tau^{2} a_{j} / a_{i}\right)
$$

\section{3 $S U(3)$ quivers - the $E_{6}$ SCFT}

We now focus on the $S U(3)$ theories. There are two kinds of non-trivial punctures: the maximal puncture, associated to the Young diagram $(3,0,0)$, which carries the full $S U(3)$ flavor symmetry; the puncture associated with the Young diagram $(2,1,0)$, which carries $U(1)$ flavor symmetry. The elementary building blocks are the 333 vertex and the 331 vertex, where 3 and 1 are shorthands for the $S U(3)$ and $U(1)$ punctures, respectively.

The 333 vertex corresponds to the $E_{6}$ SCFT of [27], denoted by $T_{3}$ in [1]. A maximal subgroup of the $E_{6}$ flavor symmetry is given by $S U(3)^{3}$ and we parametrize the Cartans of 
the three $S U(3) \mathrm{s}$ by $\mathbf{a}_{I}$. Guided by the expression of the $T_{2}$ index obtained in the previous subsection, we conjecture that the index of $T_{3}$ is given by

$$
\begin{aligned}
\mathcal{I}\left(\mathbf{a}_{\mathbf{1}}, \mathbf{a}_{\mathbf{2}}, \mathbf{a}_{\mathbf{3}}\right) & =\sum_{\lambda_{1}, \lambda_{2}} \frac{\mathcal{A}(\tau)}{P_{H L}^{\lambda_{1}, \lambda_{2}}\left(\tau^{2}, \tau^{-2}, 1 \mid \tau\right)} \prod_{I=1}^{3} \mathcal{K}\left(\mathbf{a}_{I}\right) P_{H L}^{\lambda_{1}, \lambda_{2}}\left(\mathbf{a}_{I} \mid \tau\right) \\
\mathcal{K}(\mathbf{a}) & =\frac{1}{1-\tau^{2}} \prod_{i, j=1, i \neq j}^{3} \frac{1}{\left(1-\tau^{2} a_{i} / a_{j}\right)}, \quad \prod_{i=1}^{3} a_{i}=1 \\
\mathcal{A}(\tau) & =\left(1-\tau^{4}\right)\left(1+\tau^{2}+\tau^{4}\right) .
\end{aligned}
$$

The function $\mathcal{K}(\mathbf{a})$ is fixed as always by (3.11), with $\hat{\Delta}=\Delta_{H L}$, while the overall fugacityindependent normalization factor $\mathcal{A}(\tau)$ was fixed by comparing with the known result for this index [15]. We expanded the above expression in power series in $\tau$ and found a perfect match with [15]. ${ }^{13}$ In [32] an explicit expression was conjectured for the partition function over the Higgs branch of the $E_{6}$ SCFT, which we argued in section 4 to be equivalent to the Hall-Littlewood index. This expression has a very simple form [32],

$$
\mathcal{I}\left(\mathbf{z}_{E_{6}}\right)=\sum_{k=0}^{\infty}[0, k, 0,0,0,0]_{\mathbf{z}} \tau^{2 k}
$$

where $\mathbf{z}$ is an $E_{6}$ fugacity and $[0, k, 0,0,0,0]_{\mathbf{z}}$ are the characters of the irreducible representation of $E_{6}$ with Dynkin labels $[0, k, 0,0,0,0]$. This expression is manifestly $E_{6}$ covariant while (5.23) is not: however, order by order in the $\tau$-expansion we find that the fugacities of $S U(3)^{3}$ combine to label representations of $E_{6}$ and we obtain perfect agreement. We emphasize that for this to happen the overall factors $\mathcal{K}\left(\mathbf{a}_{i}\right)$ are absolutely crucial - without taking them into account the flavor-symmetry enhancement to $E_{6}$ does not occur.

We can define an unrefined index by setting all the flavor fugacities to one. In this case the series can be easily summed up in closed form and we obtain that the unrefined index is given by

$$
\begin{aligned}
& \mathcal{I}= \\
& \frac{1+\tau^{20}+55\left(\tau^{2}+\tau^{18}\right)+890\left(\tau^{4}+\tau^{16}\right)+5886\left(\tau^{6}+\tau^{14}\right)+17929\left(\tau^{8}+\tau^{12}\right)+26060 \tau^{10}}{\left(1+\tau^{2}\right)^{-1}\left(1-\tau^{2}\right)^{22}},
\end{aligned}
$$

in complete agreement with [32].

\footnotetext{
${ }^{13}$ All the expressions for the HL index we obtain here are geometric progressions which in principle can be explicitly summed. However, for the purposes of this paper we often found it computationally more feasible and insightful to perform perturbative checks to high order in expansion in $\tau$.
} 


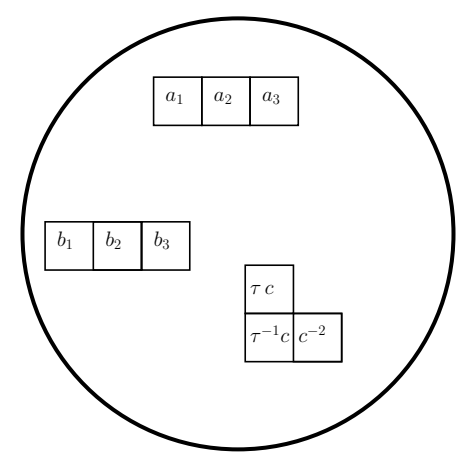

Figure 1: Association of flavor fugacities for the vertex corresponding to the 331 of the $S U(3)$ quivers. Here $a_{1} a_{2} a_{3}=1$ and $b_{1} b_{2} b_{3}=1$.

The 331 vertex corresponds to the SCFT of a free hypermultiplet in the bifundamental of $S U(3)$ and charged under $U(1)$. Its index is given by

$$
\begin{aligned}
& \mathcal{I}\left(\mathbf{a}_{1}, \mathbf{a}_{2}, c\right)=P E {\left[\tau \chi_{1}(\mathbf{a}) \chi_{1}(\mathbf{b}) c\right]_{\mathbf{a}, \mathbf{b}, c} P E\left[\tau \chi_{1}\left(\mathbf{a}^{-1}\right) \chi_{1}\left(\mathbf{b}^{-1}\right) c^{-1}\right]_{\mathbf{a}, \mathbf{b}, c} } \\
&=\prod_{i, j=1}^{3} \frac{1}{1-\tau a_{i} b_{j} c} \frac{1}{1-\tau \frac{1}{a_{i} b_{j} c}}, \quad \prod_{i=1}^{3} a_{i}=\prod_{i=1}^{3} b_{i}=1 .
\end{aligned}
$$

It can be rewritten by partially closing a puncture of the $E_{6}$ vertex (5.23), as

$$
\mathcal{I}\left(\mathbf{a}_{1}, \mathbf{a}_{2}, c\right)=\frac{1-\tau^{6}}{1-\tau^{2}} \frac{\mathcal{K}\left(\mathbf{a}_{1}\right) \mathcal{K}\left(\mathbf{a}_{2}\right)}{\left(1-\tau^{3} c^{3}\right)\left(1-\tau^{3} c^{-3}\right)} \sum_{\lambda_{1}, \lambda_{2}} \frac{P_{H L}^{\lambda_{1}, \lambda_{2}}\left(\tau c, \tau^{-1} c, c^{-2} \mid \tau\right)}{P_{H L}^{\lambda_{1}, \lambda_{2}}\left(\tau^{2}, \tau^{-2}, 1 \mid \tau\right)} \prod_{I=1}^{2} P_{H L}^{\lambda_{1}, \lambda_{2}}\left(\mathbf{a}_{I} \mid \tau\right) .
$$

The sum over representations here is a geometric progression and can be easily performed establishing the equivalence of (5.28) and (5.29) (in the process we have fixed the overall $\tau$-dependent factor).

We can use the above expressions to write the index of any $S U(3)$ quiver. Let us give again the example of the genus $\mathfrak{g}$ theory,

$$
\begin{aligned}
& \mathcal{I}_{\mathfrak{g}}^{(3)}= \\
& \left(1-\tau^{4}\right)^{\mathfrak{g}-1}\left(1-\tau^{6}\right)^{\mathfrak{g}-1}+\frac{\left(1+2\left(1+\tau^{-2}\right)^{\mathfrak{g}-1}\left(\tau^{2-2 \mathfrak{g}}-\tau^{2 \mathfrak{g}-2}\right)\right) \tau^{4(\mathfrak{g}-1)}\left(1-\tau^{2}\right)^{2 \mathfrak{g}-2}}{\left(\tau^{2-2 \mathfrak{g}}-\tau^{2 \mathfrak{g}-2}\right)^{2}} .
\end{aligned}
$$

We can subject (5.23) and (5.29) to a further non-trivial check. The channel-crossing duality of the four-punctured sphere with two $S U(3)$ and two $U(1)$ punctures corresponds to Argyres-Seiberg duality [26]. In one channel we glue together two 331 vertices along two 3 
punctures, while in the other channel the 333 vertex (index of $T_{3}$ ) is (formally) glued to a 311 vertex. Requiring equality of the two channels we find the index of the 311 vertex,

$$
\begin{array}{r}
\mathcal{I}_{311}(\mathbf{a}, c, d)=\frac{1-\tau^{6}}{\left(1-\tau^{2}\right)\left(1-\tau^{4}\right)} \frac{\mathcal{K}(\mathbf{a})}{\left(1-\tau^{3} c^{3}\right)\left(1-\tau^{3} c^{-3}\right)\left(1-\tau^{3} d^{3}\right)\left(1-\tau^{3} d^{-3}\right)} \\
\sum_{\lambda_{1}, \lambda_{2}} \frac{P_{H L}^{\lambda_{1}, \lambda_{2}}\left(\tau c, \tau^{-1} c, c^{-2} \mid \tau\right) P_{H L}^{\lambda_{1}, \lambda_{2}}\left(\tau d, \tau^{-1} d, d^{-2} \mid \tau\right) P_{H L}^{\lambda_{1}, \lambda_{2}}(\mathbf{a} \mid \tau)}{P_{H L}^{\lambda_{1}, \lambda_{2}}\left(\tau^{2}, \tau^{-2}, 1 \mid \tau\right)} .
\end{array}
$$

In the expression above the sum over representations diverges. The 311 should be regarded as a formal construct that only makes sense as a part of the larger theory. It can be interpreted as implementing a $\delta$-function constraint on the flavor indices. The non-singular way to view the gluing of 333 vertex with 311 vertex is as gauging an $S U(2)$ subgroup of $E_{6}$, as opposed to an $S U(3)$ subgroup [26]. With this interpretation of the 311 vertex, equality of the two channels amounts to

$$
\begin{aligned}
\left(1-\tau^{2}\right) & \oint \frac{d a}{4 \pi i a} P_{H L}^{\lambda_{1}, \lambda_{2}}\left(a r, a^{-1} r, r^{-2} \mid \tau\right) \prod_{\sigma_{1}, \sigma_{2}, \sigma_{3}, \sigma_{4}, \sigma_{5}= \pm 1} \frac{1}{1-\tau s^{\sigma_{1}} a^{\sigma_{2}}} \frac{1}{1-\tau^{2} r^{3 \sigma_{3}} a^{\sigma_{4}}}\left(1-a^{2 \sigma_{5}}\right) \\
& =\frac{1-\tau^{6}}{1-\tau^{4}} \frac{\prod_{\sigma= \pm 1} P_{H L}^{\lambda_{1}, \lambda_{2}}\left(\tau \frac{s^{\sigma / 3}}{r}, \tau^{-1} \frac{s^{\sigma / 3}}{r}, \frac{s^{-2 \sigma / 3}}{r^{-2}} \mid \tau\right)}{P_{H L}^{\lambda_{1}, \lambda_{2}}\left(\tau^{2}, \tau^{-2}, 1 \mid \tau\right) \prod_{\sigma_{1}, \sigma_{2}= \pm 1}\left(1-\tau^{3} s^{\sigma_{1}} / r^{3}\right)\left(1-\tau^{3} s^{\sigma_{2}} r^{3}\right)} \cdot
\end{aligned}
$$

In the first line we gauge an $S U(2)$ subgroup of $E_{6}$ and couple it to a single hypermultiplet, and in the second line a 311 vertex is glued to 333 vertex by gauging an $S U(3)$ flavor group. This is a non-trivial identity involving HL polynomials which we have checked to very high order in a perturbative expansion in $\tau$.

\subsection{A conjecture for the structure constants with generic punctures}

Extrapolating from the $S U(2)$ and $S U(3)$ cases, we are now formulate a complete conjecture for the index of all building blocks of $S U(k)$ quivers. The building blocks are classified by a triple of Young diagrams $\left(\Lambda_{1}, \Lambda_{2}, \Lambda_{3}\right)$. We conjecture

$$
\mathcal{I}_{\Lambda_{1}, \Lambda_{2}, \Lambda_{3}}=\frac{\prod_{j=2}^{k}\left(1-\tau^{2 j}\right)}{\left(1-\tau^{2}\right)^{-k-2}} \prod_{I=1}^{3} \hat{\mathcal{K}}_{\Lambda_{I}}\left(\mathbf{a}_{I}\right) \sum_{\lambda} \frac{\prod_{I=1}^{3} P_{H L}^{\lambda}\left(\mathbf{a}_{\mathbf{I}}\left(\Lambda_{I}\right) \mid \tau\right)}{P_{H L}^{\lambda}\left(\tau^{k-1}, \tau^{k-3}, \ldots, \tau^{1-k} \mid \tau\right)} .
$$

Here the assignment of fugacities according to the Young diagram labelling the type of the puncture, $\mathbf{a}(\Lambda)$, is as illustrated in figure 2. The summation over $\lambda$ is over the Young diagrams with $k-1$ rows, $\lambda=\left(\lambda_{1}, \ldots, \lambda_{k-1}\right), \lambda_{j} \geq \lambda_{j+1}$. The factors $\hat{\mathcal{K}}_{\Lambda}(\mathbf{a})$ are defined as

$$
\hat{\mathcal{K}}_{\Lambda}(\mathbf{a})=\prod_{i=1}^{\operatorname{row}(\Lambda)} \prod_{j, k=1}^{l_{i}} \frac{1}{1-\mathfrak{a}_{j}^{i} \overline{\mathfrak{a}}_{k}^{i}} .
$$

Here row $(\Lambda)$ is the number of rows in $\Lambda$ and $l_{i}$ is the length of $i$ th row. The coefficients $\mathfrak{a}_{k}^{i}$ are associated to the Young diagram as illustrated in figure 3. Our conjecture is consistent 


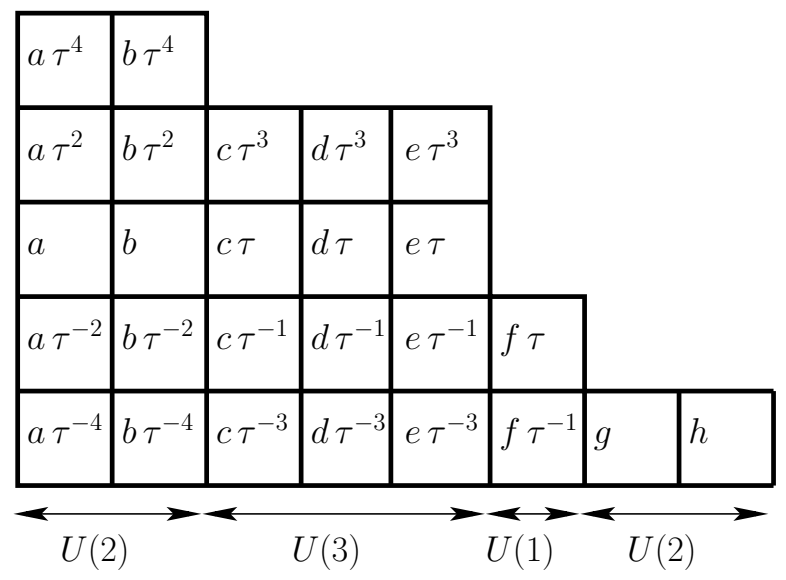

Figure 2: Association of the flavor fugacities for a generic puncture. Punctures are classified by embeddings of $S U(2)$ in $S U(k)$, so they are specified by the decomposition of the fundamental representation of $S U(k)$ into irreps of $S U(2)$, that is, by a partition of $k$. Graphically we represent the partition by an auxiliary Young diagram $\Lambda$ with $k$ boxes, read from left to right. In the figure we have the fundamental of $S U(26)$ decomposed as $\mathbf{5}+\mathbf{5}+\mathbf{4}+\mathbf{4}+\mathbf{4}+\mathbf{2}+\mathbf{1}+\mathbf{1}$. The commutant of the embedding gives the residual flavor symmetry, in this case $S(U(3) \times U(2) \times U(2) \times U(1))$, where the $S(\ldots)$ constraint amounts to removing the overall $U(1)$. The $\tau$ variable is viewed here as an $S U(2)$ fugacity, while the Latin variables are fugacities of the residual flavor symmetry. The $S(\ldots)$ constraint implies that the flavor fugacities satisfy $(a b)^{5}(c d e)^{4} f^{2} g h=1$.

with the $S U(2)$ and $S U(3)$ cases seen previously as well as with all other examples discussed below.

For three maximal punctures (the $T_{k}$ theory), (5.33) becomes

$$
\begin{aligned}
\mathcal{I}_{T_{k}}\left(\mathbf{a}_{\mathbf{1}}, \mathbf{a}_{2}, \mathbf{a}_{\mathbf{3}}\right) & =\sum_{\lambda_{1} \geq \lambda_{2} \geq \ldots \geq \lambda_{k-1}} \frac{\mathcal{A}(\tau)}{P_{H L}^{\lambda_{1}, . ., \lambda_{k-1}}\left(\tau^{k-1}, . ., \tau^{1-k} \mid \tau\right)} \prod_{I=1}^{3} \mathcal{K}\left(\mathbf{a}_{I}\right) P_{H L}^{\lambda_{1}, . . \lambda_{k-1}}\left(\mathbf{a}_{I} \mid \tau\right), \\
\mathcal{K}(\mathbf{a}) & =\frac{1}{\left(1-\tau^{2}\right)^{\frac{k-1}{2}}} \prod_{i, j=1, i \neq j}^{k} \frac{1}{\left(1-\tau^{2} a_{i} / a_{j}\right)}, \quad \prod_{i=1}^{k} a_{i}=1 \\
\mathcal{A}(\tau) & =\frac{\prod_{j=2}^{k}\left(1-\tau^{2 j}\right)}{\left(1-\tau^{2}\right)^{\frac{k-1}{2}}}
\end{aligned}
$$

Let us illustrate the power of these TQFT expressions by computing the index of the genus $\mathfrak{g} S U(k)$ theory. It is given by

$$
\mathcal{I}_{\mathfrak{g}}^{(k)}=\frac{\left(\prod_{j=2}^{k}\left(1-\tau^{2 j}\right)\right)^{2 \mathfrak{g}-2}}{\left(1-\tau^{2}\right)^{(k-1)(\mathfrak{g}-1)}} \sum_{\lambda} \frac{1}{P_{H L}^{\lambda}\left(\tau^{k-1}, \tau^{k-3}, \ldots, \tau^{1-k} \mid \tau\right)^{2 \mathfrak{g}-2}},
$$


where the summation is over all Young diagrams with $k-1$ rows, i.e. over the finite irreducible representations of $S U(k)$.

The sum over representations in (5.33) does not converge for arbitrary choices of the three Young diagrams $\Lambda_{I}$. We have already encountered an example in the last subsection: the 311 vertex of $S U(3)$ theories has a divergent expression. There is no actual SCFT corresponding to the 311 vertex, but one can glue this vertex to a larger quiver and obtain meaningful results. There are cases however where the divergent vertex cannot appear as a piece of a larger quiver and thus the expression (5.33) for its index does not have a clear physical interpretation. An example of such a vertex is the index of an $S U(6)$ theory with three $S U(3)$ punctures. We have checked in several cases that a divergence in (5.33) correlates with the fact that the graded rank of the Coulomb branch (as defined in [43]) of the putative SCFT has negative components. This is an indication that associating field theories to such punctured surfaces may be delicate. Punctured surfaces of this type were recently considered in [44] and subtleties associated with them addressed in [45].

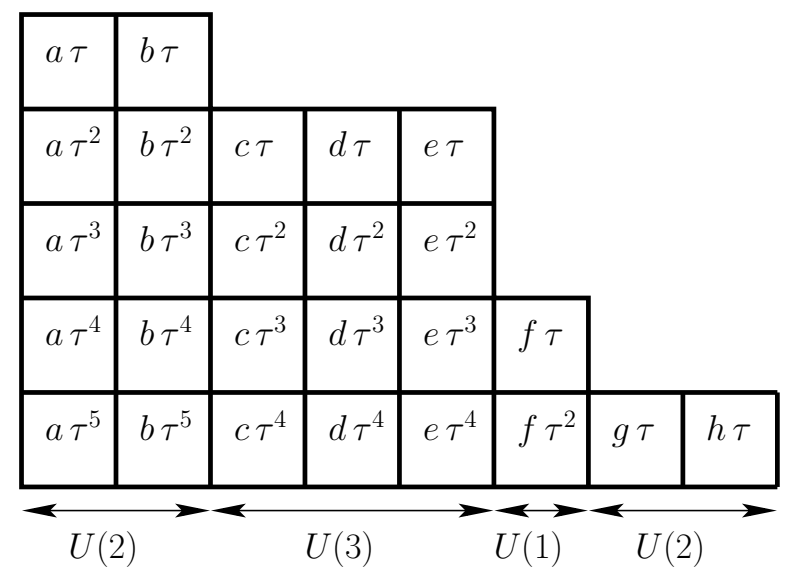

Figure 3: The factors $\mathfrak{a}_{k}^{i}$ associated to a generic Young diagram. The upper index is the row index and the lower is the column index. In $\overline{\mathfrak{a}}_{k}^{i}$ one takes the inverse of flavor fugacities while $\tau$ is treated as real number. As before, the flavor fugacities in this example satisfy $(a b)^{5}(c d e)^{4} f^{2} g h=1$.

\section{5 $S U(4)$ quivers $-T_{4}$ and the $E_{7}$ SCFT}

Let us use the general expressions of the previous section to discuss some of the features of $S U(4)$ quivers. First, from (5.35) we can compute the unrefined index of $T_{4}$,

$$
\mathcal{I}_{T_{4}}=1+45 \tau^{2}+128 \tau^{3}+1249 \tau^{4}+5504 \tau^{5}+30786 \tau^{6}+136832 \tau^{7}+623991 \tau^{8}+\ldots
$$


We present a closed form expression for it in appendix D. Refining with the flavor fugacities one gets

$$
\begin{aligned}
\mathcal{I}_{T_{4}}= & 1+[(\mathbf{1 5}, 1,1)+(1, \mathbf{1 5}, 1)+(1,1, \mathbf{1 5})] \tau^{2}+[(\mathbf{4}, \mathbf{4}, \mathbf{4})+(\overline{\mathbf{4}}, \overline{\mathbf{4}}, \overline{\mathbf{4}})] \tau^{3}+ \\
& {[1+(\mathbf{1 5}, 1,1)+(1, \mathbf{1 5}, 1)+(1,1, \mathbf{1 5})+(\mathbf{2 0}, 1,1)+(1, \mathbf{2 0}, 1)+(1,1, \mathbf{2 0})+} \\
& +(\mathbf{1 5}, \mathbf{1 5}, 1)+(1, \mathbf{1 5}, \mathbf{1 5})+(\mathbf{1 5}, 1, \mathbf{1 5})+(\mathbf{8 4}, 1,1)+(1, \mathbf{8 4}, 1)+(1,1, \mathbf{8 4})+ \\
& +(\mathbf{6}, \mathbf{6}, \mathbf{6})] \tau^{4}+\ldots
\end{aligned}
$$

In terms of Young diagrams $\mathbf{8 4}=(4,2,2), \mathbf{6}=(1,1,0), \mathbf{2 0}=(2,2,0)$. The symmetric product of the $\tau^{2}$ term reproduces all the terms at the $\tau^{4}$ order except for the $(\mathbf{6}, \mathbf{6}, \mathbf{6})$ term, and for the fact that two singlets are missing (the symmetric product contains three singlets while only one is present at order $\left.\tau^{4}\right)$. We deduce that the $(\mathbf{6}, \mathbf{6}, \mathbf{6})$ state is an additional generator of the Higgs branch, and that there is a constraint allowing only for one singlet in the symmetric product of the $\tau^{2}$ states to appear at $\tau^{4}$ order. Unlike the situation for the $E_{6}$ SCFT where the Higgs branch is generated by a single scalar transforming as $\mathbf{7 8}$ of $E_{6}$ [46] here one has new generators appearing at higher orders in the $\tau$ expansion and thus having different $E-R$ quantum numbers.

Next, we can partially close a puncture to obtain the index of the 441 vertex. On one hand, the 441 vertex correspond to the free hypermultiplet SCFT in the bifundamental of two $S U(4)$ s and charged under the $U(1)$, so its index can be evaluated by direct counting,

$$
\mathcal{I}\left(\mathbf{a}_{1}, \mathbf{a}_{2}, c\right)=\prod_{i, j}^{4} \frac{1}{1-\tau a_{i} b_{j} c} \frac{1}{1-\tau \frac{1}{a_{i} b_{j} c}}, \quad \prod_{i=1}^{4} a_{i}=\prod_{i=1}^{4} b_{i}=1 .
$$

On the other hand, from (5.33),

$$
\begin{aligned}
\mathcal{I}\left(\mathbf{a}_{1}, \mathbf{a}_{2}, c\right) & =\frac{1-\tau^{8}}{\left(1-\tau^{2}\right)} \frac{\mathcal{K}\left(\mathbf{a}_{1}\right) \mathcal{K}\left(\mathbf{a}_{2}\right)}{\left(1-\tau^{4} c^{4}\right)\left(1-\tau^{4} c^{-4}\right)} \\
\sum_{\lambda_{1}, \lambda_{2}, \lambda_{3}} & \frac{P_{H L}^{\lambda_{1}, \lambda_{2}, \lambda_{3}}\left(\tau^{2} c, c, \tau^{-2} c, c^{-3} \mid \tau\right)}{P_{H L}^{\lambda_{1}, \lambda_{2}, \lambda_{3}}\left(\tau^{3}, \tau, \tau^{-1}, \tau^{-3} \mid \tau\right)} \prod_{i=1}^{2} P_{H L}^{\lambda_{1}, \lambda_{2}, \lambda_{3}}\left(\mathbf{a}_{\mathbf{i}} \mid \tau\right) .
\end{aligned}
$$

We have checked the equivalence of these two expressions perturbatively to very high order in $\tau$.

Finally, let us look at the vertex with two maximal punctures and one puncture corresponding to a square Young diagram, which carries an $S U(2)$ flavor symmetry, see figure 4 . The flavor symmetry of this theory is known to enhance to $E_{7}$ [26]. From (5.33), the Hall-Littlewood 


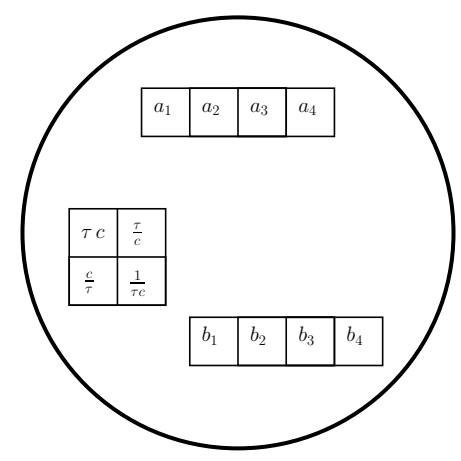

Figure 4: Association of the flavor fugacities for the $E_{7}$ vertex. Here $\prod_{i=1}^{4} b_{i}=\prod_{i=1}^{4} a_{i}=1$.

index of this SCFT is given by

$$
\begin{gathered}
\mathcal{I}_{E_{7}}\left(\mathbf{a}_{1}, \mathbf{a}_{2}, c\right)=\left(1+\tau^{2}+\tau^{4}\right)\left(1+\tau^{4}\right) \frac{\mathcal{K}\left(\mathbf{a}_{1}\right) \mathcal{K}\left(\mathbf{a}_{2}\right)}{\left(1-\tau^{2} c^{ \pm 2}\right)\left(1-\tau^{4} c^{ \pm 2}\right)} \times \\
\sum_{\lambda_{1}, \lambda_{2}, \lambda_{3}} \frac{P_{H L}^{\lambda_{1}, \lambda_{2}, \lambda_{3}}\left(\tau c, \frac{c}{\tau}, \frac{\tau}{c}, \frac{1}{\tau c} \mid \tau\right)}{P_{H L}^{\lambda_{1}, \lambda_{2}, \lambda_{3}}\left(\tau^{3}, \tau, \tau^{-1}, \tau^{-3} \mid \tau\right)} \prod_{i=1}^{2} P_{H L}^{\lambda_{1}, \lambda_{2}, \lambda_{3}}\left(\mathbf{a}_{\mathbf{i}} \mid \tau\right) .
\end{gathered}
$$

In [32] an explicit expression for the Higgs partition function was conjectured,

$$
\mathcal{I}\left(\mathbf{z}_{E_{7}}\right)=\sum_{k=0}^{\infty}[k, 0,0,0,0,0,0]_{\mathbf{z}} \tau^{2 k}
$$

where $\mathbf{z}$ is an $E_{7}$ fugacity and $[k, 0,0,0,0,0,0]_{\mathbf{z}}$ are the characters of the irreducible representation of $E_{7}$ with Dynkin labels $[k, 0,0,0,0,0,0]$. We have checked also here (5.41) is in complete agreement with [32], and thus in particular is secretly $E_{7}$ covariant: the check can be done analytically for the unrefined index and perturbatively in $\tau$ to high order for the refined one.

The expression (5.41) can be also checked by the Argyres-Seiberg duality between USp(4) theory coupled to six fundamental hypermultiplets and $E_{7}$ theory with an $S U(2)$ subgroup gauged [26]. The former has a weakly-coupled description and its index can be computed directly,

$$
\mathcal{I}_{U S p(4)}=1+\chi_{S O(12)}^{\mathbf{6 6}}(u, v, w, x, y, z) \tau^{2}+\cdots .
$$

Since there are six fundamental hypermultiplets the flavor group is $S O(12)$. On the other hand, gauging an $S U(2)$ inside one $S U(4)$ subgroup of the $E_{7}$ index (5.41) gives

$$
\mathcal{I}=\oint \frac{d e}{4 \pi i e}\left(1-e^{2}\right)\left(1-e^{-2}\right) P E\left[-\tau^{2} \chi_{2}(e)\right]_{t, e} \mathcal{I}_{E_{7}}(\mathbf{a},\{e s, s / e, b / s, 1 / b s\}, c) .
$$

We have checked perturbatively in $\tau$ that (5.43) and (5.44) coincide under the following identification of the fugacities:

$$
u \rightarrow \frac{a_{1}}{s}, \quad v \rightarrow \frac{a_{2}}{s}, \quad w \rightarrow \frac{a_{3}}{s}, \quad x \rightarrow \frac{1}{a_{1} a_{2} a_{3} s}, \quad y \rightarrow b c, \quad z \rightarrow \frac{b}{c} .
$$




\section{6 $S U(6)$ quivers - the $E_{8}$ SCFT}

As our last example, we consider the index of the $E_{8}$ SCFT [41]. This theory corresponds to a sphere with a maximal $S U(6)$ puncture and two non-maximal punctures with $S U(3)$ and $S U(2)$ flavor symmetries, see figure 5. The group $S U(6) \times S U(3) \times S U(2)$ is a maximal subgroup of $E_{8}$. Following the general prescription (5.33) the index of $E_{8}$ SCFT is given by

$$
\begin{aligned}
& \mathcal{I}_{E_{8}}\left(\mathbf{a}, b_{1}, b_{2}, c\right)=\frac{\left(1-\tau^{8}\right)\left(1-\tau^{10}\right)\left(1-\tau^{12}\right)}{\left(1-\tau^{2}\right)^{1 / 2}\left(1-\tau^{4}\right)^{4}\left(1-\tau^{6}\right)} \times \\
& \frac{\mathcal{K}(\mathbf{a})}{\left(1-\tau^{2} c^{ \pm 2}\right)\left(1-\tau^{4} c^{ \pm 2}\right)\left(1-\tau^{6} c^{ \pm 2}\right) \prod_{i \neq j}\left(1-\tau^{2} b_{i} / b_{j}\right)\left(1-\tau^{4} b_{i} / b_{j}\right)} \times \\
& \sum_{\lambda_{1}, \ldots, \lambda_{5} \equiv \lambda} \frac{P_{H L}^{\lambda}\left(\tau b_{1}, \tau b_{2}, \tau b_{3}, \frac{b_{1}}{t}, \frac{b_{2}}{\tau}, \frac{b_{3}}{\tau} \mid \tau\right) P_{H L}^{\lambda}\left(\tau^{2} c, c, \frac{c}{\tau^{2}}, \frac{\tau^{2}}{c}, \frac{1}{c}, \frac{1}{\tau^{2} c}, \mid \tau\right) P_{H L}^{\lambda}\left(\mathbf{a}_{\mathbf{i}} \mid \tau\right)}{P_{H L}^{\lambda}\left(\tau^{5}, \tau^{3}, \tau, \tau^{-1}, \tau^{-3}, \tau^{-5} \mid \tau\right)} .
\end{aligned}
$$

In [32] it was conjectured that the Higgs partition function has the following $E_{8}$ covariant expansion,

$$
\mathcal{I}\left(\mathbf{z}_{E_{8}}\right)=\sum_{k=0}^{\infty}[k, 0,0,0,0,0,0,0]_{\mathbf{z}} \tau^{2 k},
$$

where $\mathbf{z}$ is an $E_{8}$ fugacity and $[k, 0,0,0,0,0,0,0]_{\mathbf{z}}$ are the characters of the irreducible representation of $E_{8}$ with Dynkin labels $[k, 0,0,0,0,0,0,0]$. We have again checked equivalence of (5.46) and (5.47) in the $\tau$-expansion, though in this case due to computational complexity we could perform the expansion only up to order $\tau^{8}$. The size of representations of $E_{8}$ contributing to the index grows very fast with the order of $\tau, e . g$. the unrefined index is given by

$$
\mathcal{I}_{E_{8}}=1+248 \tau^{2}+27000 \tau^{4}+1763125 \tau^{6}+79143000 \tau^{8}+\ldots
$$

\subsection{Large $k$ limit}

It is not difficult to evaluate the large $k$ limit of the HL index of $S U(k)$ generalized quivers. ${ }^{14}$ For instance, for the index of the theory corresponding to a genus $\mathfrak{g}$ surface without punctures (5.36),

$$
\mathcal{I}_{\mathfrak{g}}^{(k \rightarrow \infty)}=\prod_{j=2}^{\infty}\left(1-\tau^{2 j}\right)^{\mathfrak{g}-1}=P E\left[-(\mathfrak{g}-1) \frac{\tau^{4}}{1-\tau^{2}}\right] .
$$

\footnotetext{
${ }^{14}$ We thank Davide Gaiotto and Juan Maldacena for discussions on issues related to this section.
} 


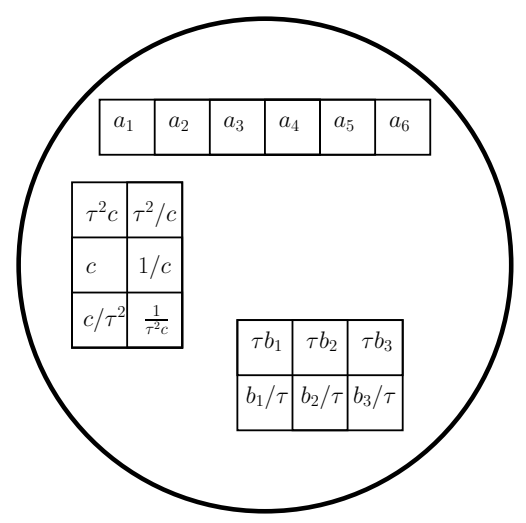

Figure 5: Association of the flavor fugacities for the $E_{8}$ vertex. Here $\prod_{i=1}^{3} b_{i}=\prod_{i=1}^{6} a_{i}=1$.

In appendix $\mathrm{C}$ we give a short derivation of this expression. In the large $k$ limit only the singlet in the sum over the representations of (5.36) contributes. Since (5.49) is of order one for large $k$ it is expected to be matched by counting the appropriate supergravity modes in the dual AdS background [47]. We can also compute the index of the $T_{k}$ theories in the large $k$ limit,

$$
\begin{aligned}
\mathcal{I}_{T_{k \rightarrow \infty}}\left(\mathbf{a}_{\mathbf{1}}, \mathbf{a}_{2}, \mathbf{a}_{3}\right) & =\prod_{j=2}^{\infty} \frac{1}{1-\tau^{2 j}} \prod_{I=1}^{3} \prod_{j \neq i}^{\infty} \frac{1}{1-\tau^{2} a_{i}^{I} / a_{j}^{I}} \\
& =P E\left[\frac{\tau^{4}}{1-\tau^{2}}\right] \prod_{I=1}^{3} P E\left[\tau^{2} \sum_{i \neq j} a_{i}^{I} / a_{j}^{I}\right]
\end{aligned}
$$

From here the large $k$ index of any generalized quiver is trivial to compute; in particular (5.49) can be obtained by gluing together the index of (5.50). Unrefining the index of $T_{k}$ by setting all the flavor fugacities $a_{j}^{I}=1$ we see that it has a non-trivial $k$ dependence for large $k$ limit. Taking the plethystic log of (5.50) (that is, considering the index of single-particle states) we find

$$
\mathcal{I}_{T_{k \rightarrow \infty}}^{s . p .}=3 \tau^{2}\left(k^{2}-k\right)+\frac{\tau^{4}}{1-\tau^{2}}+O\left(\frac{1}{k}\right)
$$

The term of order $k^{2}$ on the right-hand-side comes from states in the adjoint representation of the flavor group, while the term of order $k$ comes from neutral states. At least some of $O\left(k^{2}\right)$ states in the adjoint representation must correspond to modes of the AdS gauge fields that couple to the flavor currents of the boundary theory. It would be interesting to check whether all the $O\left(k^{2}\right)$ and $O(k)$ states can be accounted for by supergravity states. If not, the extra states could arise as non-perturbative states in the bulk geometry (e.g. wrapped branes or black holes). In all cases studied so far the index is of order one in the large $k$ limit and thus 
cannot capture the non-perturbative states of the bulk theory $[12,48,49,23]$. This is not a contradiction, since the index only counts protected states with signs. The index vanishes on combinations of short multiplets that can in principle recombine into long ones, even when such kinematically-allowed recombination do not actually happen [12]. However, for linear quivers (in particular for the $T_{k}$ theories) the HL index has the meaning of a Hilbert series over the Higgs branch, so it is expected to capture all the relevant $\frac{3}{8}$-BPS states of the dual theory. We leave the very interesting comparison with the bulk theory for future research.

\section{Schur index}

We turn to the Schur index,

$$
\mathcal{I}_{S}=\operatorname{Tr}(-1)^{F} q^{E-R}
$$

which is the same as the reduced index considered in [16]. Let us first recall the expression for the $S U(k)$ propagator. It is of the usual form

$$
\eta\left(\mathbf{a}, \mathbf{b}^{-1}\right)=\Delta(\mathbf{a}) \mathcal{I}^{V}(\mathbf{a}) \delta\left(\mathbf{a}, \mathbf{b}^{-1}\right)
$$

where $\Delta(\mathbf{a})$ is the Haar measure (5.21), and $\mathcal{I}^{V}(\mathbf{a})$ the index of the vector multiplet, given by

$$
\mathcal{I}_{q}^{V}(\mathbf{a})=P E\left[\frac{-2 q}{1-q} \chi_{a d j}(\mathbf{a})\right]_{q, \mathbf{a}} .
$$

The set of functions $\left\{f_{q}^{\lambda}(\mathbf{a})\right\}$ that diagonalize the structure constants are proportional to the Schur polynomials [16],

$$
f_{q}^{\lambda}(\mathbf{a})=\mathcal{K}_{q}(\mathbf{a}) \chi^{\lambda}(\mathbf{a})
$$

The Schur polynomials are orthonormal under the Haar measure, so in this case $\hat{\Delta}(\mathbf{a})=\Delta(\mathbf{a})$ (recall (3.10)) and the factor $\mathcal{K}_{q}(\mathbf{a})$ is given by

$$
\mathcal{K}_{q}(\mathbf{a})=\frac{1}{\left[\mathcal{I}_{q}^{V}(\mathbf{a})\right]^{\frac{1}{2}}} .
$$

Generalizing our results in [16], we conjecture the following expression for the Schur index of a three-punctured sphere with generic punctures,

$$
\mathcal{I}_{\Lambda_{1}, \Lambda_{2}, \Lambda_{3}}=\frac{(q ; q)^{k+2}}{\prod_{j=1}^{k-1}\left(1-q^{j}\right)^{k-j}} \prod_{I=1}^{3} \hat{\mathcal{K}}_{\Lambda_{I}}\left(\mathbf{a}_{I}\right) \sum_{\lambda} \frac{\prod_{I=1}^{3} \chi^{\lambda}\left(\mathbf{a}_{\mathbf{I}}\left(\Lambda_{I}\right)\right)}{\chi^{\lambda}\left(q^{\frac{k-1}{2}}, q^{\frac{k-3}{2}}, \ldots, q^{\frac{1-k}{2}}\right)}
$$


Here the sum is over the finite-dimensional irreducible representations of $S U(k)$. The assignment of fugacities according to the Young diagram, a $(\Lambda)$, is again as in figure 2, with $\tau \rightarrow q^{1 / 2}$. The Pochhammer symbol $(a ; b)$ is defined by

$$
(a ; b)=\prod_{i=0}^{\infty}\left(1-a b^{i}\right) .
$$

The character of the representation corresponding to Young diagram $\lambda=\left(\lambda_{1}, \ldots, \lambda_{k-1}, 0\right)$ is given by a Schur polynomial,

$$
\chi^{\lambda}(\mathbf{a})=\frac{\operatorname{det}\left(a_{i}^{\lambda_{j}+k-j}\right)}{\operatorname{det}\left(a_{i}^{k-j}\right)} .
$$

The $\hat{\mathcal{K}}_{\Lambda}$ prefactors are given by

$$
\hat{\mathcal{K}}_{\Lambda}(\mathbf{a})=\prod_{i=1}^{\operatorname{row}(\Lambda)} \prod_{j, k=1}^{l_{i}} P E\left[\frac{\mathfrak{a}_{j}^{i} \overline{\mathfrak{a}}_{k}^{i}}{1-q}\right]_{\mathfrak{a}_{i}, q},
$$

where $\operatorname{row}(\Lambda)$ is the number of rows in $\Lambda$ and $l_{i}$ is the length of $i$ th row. The coefficients $\mathfrak{a}_{k}^{i}$ are associated to the Young diagram again as in figure 3, with $\tau \rightarrow q^{1 / 2}$. Note that the quantity appearing in the denominator of (6.6) is the quantum dimension of the representation $\lambda$ of $S U(k)$,

$$
\operatorname{dim}_{q} \lambda=\chi^{\lambda}\left(q^{\frac{k-1}{2}}, q^{\frac{k-3}{2}}, \ldots, q^{\frac{1-k}{2}}\right)
$$

For $S U(2)$ the quantum dimension is also known as the $q$-number $[\lambda]_{q}$.

We have subjected (6.6) to similar checks as the one described for the Hall-Littlewood index, finding complete agreement with expectations; a few such checks were reported in [16]. Let us only mention here the basic identity following from compatibility of (6.6) with the index of the $S U(2)$ trifundamental hypermultiplet,

$$
\begin{aligned}
P E & {\left[\frac{q^{1 / 2}}{1-q}\left(a_{1}+\frac{1}{a_{1}}\right)\left(a_{2}+\frac{1}{a_{2}}\right)\left(a_{3}+\frac{1}{a_{3}}\right)\right]_{a_{i}, \tau}=} \\
& (q ; q)^{3}\left(q^{2} ; q\right) \prod_{i=1}^{3} P E\left[\frac{q}{1-q}\left(a_{i}^{2}+a_{i}^{-2}+2\right)\right]_{a_{i}, \tau} \sum_{\lambda=0}^{\infty} \frac{\prod_{i=1}^{3} \chi^{\lambda}\left(a_{i}, a_{i}^{-1}\right)}{\chi^{\lambda}\left(q^{\frac{1}{2}}, q^{-\frac{1}{2}}\right)}
\end{aligned}
$$

A proof of this identity is outlined in appendix E. 


\section{Macdonald index}

We are now ready to combine and generalize the results of the two previous sections. The HallLittlewood and Schur polynomials are special cases of a two-parameter family of polynomials discovered by Macdonald [42]. One naturally expects Macdonald polynomials to be relevant for the calculation of the index in a two-dimensional slice of the full three-dimensional fugacity space. The precise confirmation of this idea is our main result. Identifying the correct slice is by no means obvious, but at this point it will come as no great surprise that it is given by the limit that we have called the Macdonald index in section 4,

$$
\mathcal{I}_{M}=\operatorname{Tr}_{M}(-1)^{F} q^{E-2 R-r} t^{R+r}=\operatorname{Tr}_{M}(-1)^{F} q^{-2 j_{1}} t^{R+r}
$$

where $\operatorname{Tr}_{M}$ denotes the trace restricted to states with $\delta_{1+}=E+2 j_{1}-2 R-r=0$. For $q=t$ Macdonald polynomials reduce to Schur polynomials, while for $q=0$ they reduce to Hall-Littlewood polynomials. By design, the Macdonald trace formula (7.1) reproduces respectively the Schur and Hall-Littlewood trace formulae in the same limits.

Our basic ansatz is that the complete set of functions $\left\{f_{q, t}^{\lambda}(\mathbf{a})\right\}$ that diagonalize the structure constants are proportional to Macdonald polynomials with parameters $q$ and $t$,

$$
f_{q, t}^{\lambda}(\mathbf{a})=\mathcal{K}_{q, t}(\mathbf{a}) P^{\lambda}(\mathbf{a} \mid q, t)
$$

The Macdonald polynomials [42] ${ }^{15}\left\{P^{\lambda}(\mathbf{a})\right\}$ are defined as the set of polynomials labeled by Young diagrams $\lambda$, orthonormal under the measure

$$
\Delta_{q, t}(\mathbf{a})=\frac{1}{k !} P E\left[-\frac{1-t}{1-q}\left(\chi_{a d j}(\mathbf{a})-k+1\right)\right]_{q, t, \mathbf{a}}=\frac{1}{k !} \prod_{n=0}^{\infty} \prod_{i \neq j} \frac{1-q^{n} a_{i} / a_{j}}{1-t q^{n} a_{i} / a_{j}},
$$

and having the expansion

$$
P^{\lambda}=\mathcal{N}_{\lambda}(q, t)\left\{m_{\lambda}+\sum_{\mu<\lambda} h_{\lambda \mu}(q, t) m_{\mu}\right\}
$$

Here we define

$$
m_{\lambda=\left(\lambda_{1}, . ., \lambda_{k}\right)}(\mathbf{a})=\sum_{\sigma \in S_{k}^{\prime}} \prod_{i=1}^{k} a_{i}^{\sigma\left(\lambda_{i}\right)}
$$

where $S_{k}^{\prime}$ denotes the set of distinct permutations of $\left(\lambda_{1}, \ldots, \lambda_{k}\right)$.

\footnotetext{
${ }^{15}$ Macdonald polynomials appear in physics in many different contexts. Some recent papers on subjects related to $\mathcal{N}=2$ gauge theories that discuss Macdonald polynomials are [50, 51, 52].
} 
The factor $\mathcal{K}_{q, t}(\mathbf{a})$ is again fixed by requiring orthonormality of $\left\{f_{q, t}^{\lambda}(\mathbf{a})\right\}$ under the propagator measure. The propagator takes the standard form

$$
\eta\left(\mathbf{a}, \mathbf{b}^{-1}\right)=\Delta(\mathbf{a}) \mathcal{I}^{V}(\mathbf{a}) \delta\left(\mathbf{a}, \mathbf{b}^{-1}\right),
$$

where as always $\Delta(\mathbf{a})$ is the Haar measure (5.21), while the index of the vector multiplet is in this case given by

$$
\mathcal{I}_{q, t}^{V}(\mathbf{a})=P E\left[\frac{-q-t}{1-q} \chi_{a d j}(\mathbf{a})\right]_{q, \mathbf{a}}
$$

We then have

$$
\mathcal{K}_{q, t}(\mathbf{a})=\left(\frac{\Delta_{q, t}(\mathbf{a})}{\Delta(\mathbf{a}) \mathcal{I}_{q, t}^{V}(\mathbf{a})}\right)^{\frac{1}{2}}
$$

We can finally state our main conjecture. The Macdonald index of the $S U(k)$ quiver theory associated to a sphere with three punctures of generic type is

$$
\mathcal{I}_{\Lambda_{1}, \Lambda_{2}, \Lambda_{3}}=(t ; q)^{k+2} \prod_{j=2}^{k} \frac{\left(t^{j} ; q\right)}{(q ; q)} \prod_{I=1}^{3} \hat{\mathcal{K}}_{\Lambda_{I}}\left(\mathbf{a}_{I}\right) \sum_{\lambda} \frac{\prod_{I=1}^{3} P^{\lambda}\left(\mathbf{a}_{I}\left(\Lambda_{I}\right) \mid q, t\right)}{P^{\lambda}\left(t^{\frac{k-1}{2}}, t^{\frac{k-3}{2}}, \ldots, t^{\frac{1-k}{2}} \mid q, t\right)} .
$$

The assignment of fugacities according to the Young diagram $\mathbf{a}_{\mathbf{i}}\left(\Lambda_{i}\right)$ is again as in figure 2 , with $\tau \rightarrow t^{1 / 2}$. The $\hat{\mathcal{K}}$ prefactors are

$$
\hat{\mathcal{K}}_{\Lambda}(\mathbf{a})=\prod_{i=1}^{\operatorname{row}(\Lambda)} \prod_{j, k=1}^{l_{i}} P E\left[\frac{\mathfrak{a}_{j}^{i} \overline{\mathfrak{a}}_{k}^{i}}{1-q}\right]_{\mathfrak{a}_{i}, q}
$$

with the coefficients $\mathfrak{a}_{k}^{i}$ associated to the Young diagram again as in figure 3 , with $\tau \rightarrow t^{1 / 2}$. It is immediate to check that (7.9) reduces to the HL and Schur expressions in the respective limits. For three maximal punctures (7.9) becomes,

$$
\begin{aligned}
\mathcal{I}_{T_{k}}\left(\mathbf{a}_{\mathbf{1}}, \mathbf{a}_{\mathbf{2}}, \mathbf{a}_{\mathbf{3}}\right) & =\sum_{\lambda_{1} \geq \lambda_{2} \geq \ldots \geq \lambda_{k-1}} \frac{\mathcal{A}(q, t)}{P^{\lambda_{1}, . ., \lambda_{k-1}}\left(t^{\frac{k-1}{2}}, . ., t^{\frac{1-k}{2}} \mid q, t\right)} \prod_{I=1}^{3} \mathcal{K}_{q, t}\left(\mathbf{a}_{I}\right) P^{\lambda_{1}, . . \lambda_{k-1}}\left(\mathbf{a}_{I} \mid q, t\right), \\
\mathcal{A}(q, t) & =P E\left[\frac{1}{2}(k-1) \frac{t-q}{1-q}\right] \prod_{j=2}^{k}\left(t^{j} ; q\right) .
\end{aligned}
$$

For $k=2$, this expression must agree with the index of the hypermultiplet in the trifundamental representation of $S U(2)$,

$$
\begin{aligned}
P E & {\left[\frac{t^{1 / 2}}{1-q}\left(a_{1}+\frac{1}{a_{1}}\right)\left(a_{2}+\frac{1}{a_{2}}\right)\left(a_{3}+\frac{1}{a_{3}}\right)\right]_{a_{i}, q, t}=} \\
& \frac{(t ; q)^{4}\left(t^{2} ; q\right)}{(q ; q)} \prod_{i=1}^{3} P E\left[\frac{t}{1-q}\left(a_{i}^{2}+a_{i}^{-2}+2\right)\right]_{a_{i}, q, t} \sum_{\lambda=0}^{\infty} \frac{\prod_{i=1}^{3} P^{\lambda}\left(a_{i}, a_{i}^{-1} \mid q, t\right)}{P^{\lambda}\left(t^{\frac{1}{2}}, t^{-\frac{1}{2}} \mid q, t\right)} .
\end{aligned}
$$


We have verified this identity in the $t$ and $q$ expansions. It helps that for $S U(2)$ one can write an explicit form for the Macdonald polynomials,

$$
P^{\lambda}\left(a, a^{-1} \mid q, t\right)=\mathcal{N}_{\lambda}(q, t) \sum_{i=0}^{\lambda} \prod_{j=0}^{i-1} \frac{1-t q^{j}}{1-q^{j+1}} \prod_{j=0}^{\lambda-i-1} \frac{1-t q^{j}}{1-q^{j+1}} a^{2 i-\lambda}
$$

where $\mathcal{N}_{\lambda}(q, t)$ is a normalization constant rendering the Macdonald polynomials orthonormal under the measure (7.3). More generally, equating the index for the (nn1) vertex from (7.9) with the index of a hypermultiplet in the bifundamental representation of $S U(k)$ and charged under $U(1)$, we obtain the identity

$$
\begin{aligned}
& P E\left[\frac{t^{1 / 2}}{1-q}\left(c \sum_{i, j=1}^{k} a_{i} b_{j}+\frac{1}{c} \sum_{i, j=1}^{k} a_{i}^{-1} b_{j}^{-1}\right)\right]_{a, b, c, q, t}=\frac{(t ; q)^{k}}{(q ; q)^{k-1}}\left(t^{k} ; q\right) \times \\
& P E\left[\frac{t}{1-q} \sum_{i, j=1}^{k} a_{i} a_{j}^{-1}\right]_{a, q, t} P E\left[\frac{t}{1-q} \sum_{i, j=1}^{k} b_{i} b_{j}^{-1}\right]_{b, q, t} P E\left[\frac{t^{\frac{k}{2}}}{1-q}\left(c^{k}+c^{-k}\right)\right]_{c, q, t} \times \\
& \sum_{\lambda} \frac{P^{\lambda}\left(c t^{\frac{k-2}{2}}, c t^{\frac{k-4}{2}}, \ldots, c t^{\frac{2-k}{2}}, c^{1-k} \mid q, t\right) P^{\lambda}\left(a_{i} \mid q, t\right) P^{\lambda}\left(b_{i} \mid q, t\right)}{P^{\lambda}\left(t^{\frac{k-1}{2}}, t^{\frac{k-3}{2}}, \ldots, t^{\frac{1-k}{2}} \mid q, t\right)} .
\end{aligned}
$$

It would be interesting to have an analytic proof of these identities.

From (3.12) we can readily calculate the index of the genus $\mathfrak{g}$ theory with $s$ punctures,

$$
\mathcal{I}_{\mathfrak{g}, s}\left(\mathbf{a}_{I} ; q, t\right)=\prod_{j=2}^{k}\left(t^{j} ; q\right)^{2 \mathfrak{g}-2+s} \frac{(t ; q)^{(k-1)(1-\mathfrak{g})+s}}{(q ; q)^{(k-1)(1-\mathfrak{g})}} \sum_{\lambda} \frac{\prod_{i=1}^{s} \hat{\mathcal{K}}_{\Lambda_{i}}\left(\mathbf{a}_{i}\right) P^{\lambda}\left(\mathbf{a}_{i}\left(\Lambda_{i}\right) \mid q, t\right)}{\left[P^{\lambda}\left(t^{\frac{k-1}{2}}, t^{\frac{k-3}{2}}, \ldots, t^{\frac{1-k}{2}} \mid q, t\right)\right]^{2 \mathfrak{g}-2+s}}
$$

Let us dwell upon this result. Let us first consider the genus $\mathfrak{g}$ partition function (no punctures) in the Schur limit, $q=t$. We can write it as

$$
\mathcal{I}_{\mathfrak{g}}(q)=\left[(q ; q)^{2 \mathfrak{g}-2}\right]^{k-1} S_{00}(q)^{2-2 \mathfrak{g}} \sum_{\lambda} \frac{1}{\left[\operatorname{dim}_{q}(\lambda)\right]^{2 \mathfrak{g}-2}}
$$

Here $S_{00}$ is the partition function of $S U(k)$ level $\ell$ Chern-Simons theory on $S^{3}$ if we formally identify $q=e^{\frac{2 \pi i}{\ell+k}}$,

$$
S_{00}(q)=\prod_{j=2}^{k} \frac{(q ; q)}{\left(q^{j} ; q\right)}
$$

The expression (7.16), up to the simple factor $\left[(q ; q)^{2 \mathfrak{g}-2}\right]^{k-1}$, is the genus $\mathfrak{g}$ partition function of $q$-deformed $2 d$ Yang-Mills theory in the zero area limit [28], which is in fact the same as 


\begin{tabular}{|l|l|l|}
\hline Symbol & Surface & Value \\
\hline \hline$C_{\alpha \beta \gamma}$ & \\
\hline & \\
\hline
\end{tabular}

Table 3: The structure constants, the cap, and the metric for the TQFT of the Macdonald index.

the partition function of $S U(k)$ level $\ell$ Chern-Simons theory on $\mathcal{C}_{\mathfrak{g}} \times S^{1}$ with $q=e^{\frac{2 \pi i}{\ell+k}}[28] .{ }^{16}$ If we reintroduce punctures, the index is related to a correlator the $q$-deformed $2 d$ Yang-Mills theory; the relation involving both a flavor independent factor and flavor-dependent factors $\hat{\mathcal{K}}_{\Lambda}$ associated to the punctures. We have recovered in more generality the relation found in [16] between the Schur index and $2 d q$-deformed Yang-Mills theory. ${ }^{17}$

In the more general case of $q \neq t$ the genus $\mathfrak{g}$ partition function can be written as

$$
\mathcal{I}_{\mathfrak{g}}(q, t)=\left[(t ; q)^{\mathfrak{g}-1}(q ; q)^{\mathfrak{g}-1}\right]^{k-1} \hat{S}_{00}(q, t)^{2-2 \mathfrak{g}} \sum_{\lambda} \frac{1}{\left[\operatorname{dim}_{q, t}(\lambda)\right]^{2 \mathfrak{g}-2}},
$$

where the generalized quantum dimension is given by

$$
\operatorname{dim}_{q, t}(\lambda)=P^{\lambda}\left(t^{\frac{k-1}{2}}, t^{\frac{k-3}{2}}, \ldots, t^{\frac{1-k}{2}} \mid q, t\right)
$$

\footnotetext{
${ }^{16}$ More precisely, $q$-deformed Yang-Mills theory in the zero area limit can be viewed as an analytical continuation of Chern-Simons theory, or equivalently of the G/G WZW model (see [53] for a review of the latter), to non-integer rank $\ell$.

${ }^{17}$ Ordinary $2 d$ Yang-Mills theory [36, 37] is obtained by sending $q \rightarrow 1$. From the index perspective, because of the additional overall factors, this is a singular limit. However, with proper regularization this limit can be understood as reducing the $4 d$ index to a $3 d$ partition function $[54,55,56]$. See also [57] for yet another $3 \mathrm{~d} / 4 \mathrm{~d}$ connection.
} 
and we have defined

$$
\hat{S}_{00}(q, t)=\prod_{j=2}^{k} \frac{(t ; q)}{\left(t^{j} ; q\right)} .
$$

This result appears to be closely related to the refinement of Chern-Simons theory recently discussed by Aganagic and Shakirov [31]. Up to overall factors, $\mathcal{I}_{\mathfrak{g}}(q, t)$ is equal to the partition function of refined Chern-Simons on $\mathcal{C}_{\mathfrak{g}} \times S^{1}$. In terms of the Chern-Simons matrix model the refinement of [31] amounts to changing the matrix integral measure from Haar to Macdonald. We can thus identify the $2 d$ theory whose correlators give the Macdonald index as the theory obtained from $q$-Yang-Mills theory by deforming in the same way the path integral measure. It would be interesting to find a more conventional Lagrangian description of this $2 d$ theory, for example the deformed measure could arise by integrating out some matter fields. It would also be desirable to have a better understanding of the flavor-independent factors needed to relate $2 d$ Yang-Mills ( $q$-deformed or $(q, t)$-deformed) to the index. They can be formally associated to a decoupled TQFT with a single operator (the identity). Perhaps this decoupled TQFT plays a similar role as the decoupled $U(1)$ factor in the AGT correspondence [7].

\section{Coulomb-branch index}

Finally we consider the index

$$
\mathcal{I}_{C M}(T, Q)=\operatorname{Tr}_{C M}(-1)^{F} T^{\frac{1}{2}\left(E+2 j_{1}-2 R-r\right)} Q^{\frac{1}{2}\left(E+2 j_{2}+2 R+r\right)},
$$

where $\operatorname{Tr}_{C M}$ stands for the trace over states with $E+2 j_{1}+r=0$. This limit of the full index makes sense for theories with a Lagrangian description, since the single-letter partition functions have well-defined expressions,

$$
f^{\frac{1}{2} H}=0, \quad f^{V}=\frac{T-Q}{1-Q} .
$$

Theories connected to Lagrangian theories by dualities also have a well-defined $\mathcal{I}_{C M}(T, Q)$. As discussed in section 4 , the further limit $Q \rightarrow 0$ leads to the $\mathcal{I}_{C}(T)$ index, which is guaranteed to be well-defined for any $\mathcal{N}=2 \mathrm{SCFT}$.

We refer to (8.1) as the "Coulomb-branch" index, or Coulomb index for short, because in a Lagrangian theory it receives contributions only from the $\overline{\mathcal{E}}$-type short-multiplets (see Appendix B), whose bottom components are the gauge-invariant operators that parametrize the Coulomb branch, for example

$$
\operatorname{Tr} \phi^{2}, \operatorname{Tr} \phi^{3}, \ldots, \operatorname{Tr} \phi^{k}
$$

for a theory with $S U(k)$ gauge group. Since the hypermultiplets do not contribute, the Coulomb index is independent of the flavor fugacities and the TQFT structure is very simple. 
The structure constants associated to a three-punctured sphere depend only on $T$ and $Q$, and so does the propagator, since the gauge-group matrix integral can be carried out independently of what the propagator connects to. The index of a quiver is then just a product over the indices of its constituents (propagators and vertices).

The index of a vector multiplet in the adjoint representation of a simple gauge group $\mathcal{G}$ is

$$
\mathcal{I}_{(\mathcal{G})}^{V}(Q, T)=\oint_{\mathbb{T}^{r} \mathcal{G}} \prod_{i=1}^{r_{\mathcal{G}}} \frac{d a_{i}}{2 \pi i} \Delta_{\mathcal{G}}(\mathbf{a}) \exp \left[-\sum_{n=1}^{\infty} \frac{1}{n} \frac{Q^{n}-T^{n}}{1-Q^{n}} \chi_{a d j}^{(\mathcal{G})}\left(\mathbf{a}^{n}\right)\right]
$$

where $r_{\mathcal{G}}$ is the rank of $\mathcal{G}$ and $\Delta_{\mathcal{G}}(\mathbf{a})$ the Haar measure,

$$
\Delta_{\mathcal{G}}(\mathbf{a})=\frac{1}{\left|W_{\mathcal{G}}\right|} \exp \left[-\sum_{n=1}^{\infty} \frac{1}{n}\left(\chi_{a d j}\left(\mathbf{a}^{n}\right)-r_{\mathcal{G}}\right)\right]
$$

with $\left|W_{\mathcal{G}}\right|$ the order of the Weyl group. We recognize the integrand in (8.4) as the Macdonald measure (7.3) with parameters $Q$ and $T$. The integral can be evaluated explicitly thanks to Macdonald's celebrated constant-term identities (see e.g. $[42,58]$ for pedagogic expositions and [59] for a brief review),

$$
\mathcal{I}_{\mathcal{G}}^{V}=P E\left[\operatorname{rank}(\mathcal{G}) \tilde{\mathcal{I}}_{1}+\sum_{\alpha \in R^{+}} \tilde{\mathcal{I}}_{1+\mathcal{C}_{\alpha}}-\tilde{\mathcal{I}}_{\mathcal{C}_{\alpha}}\right], \quad \mathcal{C}_{\alpha} \equiv \sum_{\beta \in R^{+}} \frac{(\alpha, \beta)}{(\beta, \beta)},
$$

where $R^{+}$is the collection of positive roots of $\mathcal{G}$ and

$$
\tilde{\mathcal{I}}_{\ell}=T^{\ell-1} \frac{T-Q}{1-Q} .
$$

We recognize $\tilde{\mathcal{I}}_{\ell}$ as the index of the $\overline{\mathcal{E}}_{-\ell(0,0)}$ superconformal multiplet, which satisfies the shortening condition $E=\ell$ (see appendix B). By a Lie-algebraic identity, (8.6) can be rewritten more succinctly as [59]

$$
\mathcal{I}_{\mathcal{G}}^{V}=P E\left[\sum_{j \in \exp (\mathcal{G})} \tilde{\mathcal{I}}_{j+1}\right]
$$

where $\exp (\mathcal{G})$ stands for the set of exponents of the Lie group $\mathcal{G}$. This result has an immediate physical interpretation. The Coulomb index is saturated by the $\overline{\mathcal{E}}$-multiplets, whose bottom components are the gauge-invariant operators made of $\phi s$. The single-particle index (the argument of the plethystic exponential in (8.8)) then counts the independent gauge-invariant operators made of $\phi \mathrm{s}$, which are in 1-1 correspondence with the Casimirs of the group, that is with $\exp (\mathcal{G})$. For example, for $\mathcal{G}=S U(k), \exp (\mathcal{G})=\{1,2, \ldots, k-1\}$, and we see that the Coulomb index counts the independent single-trace operators (8.3) that parametrize the 
Coulomb branch. Turning the logic around, we can view this as a "physical" (or perhaps, combinatorial) proof of Macdonald's constant term identities. The integral over the Macdonald measure (8.4) counts gauge-invariant words built from certain letters of the vector multiplet; from superconformal representation theory we can identify which short multiplets are relevant for this counting problem, and deduce (8.8).

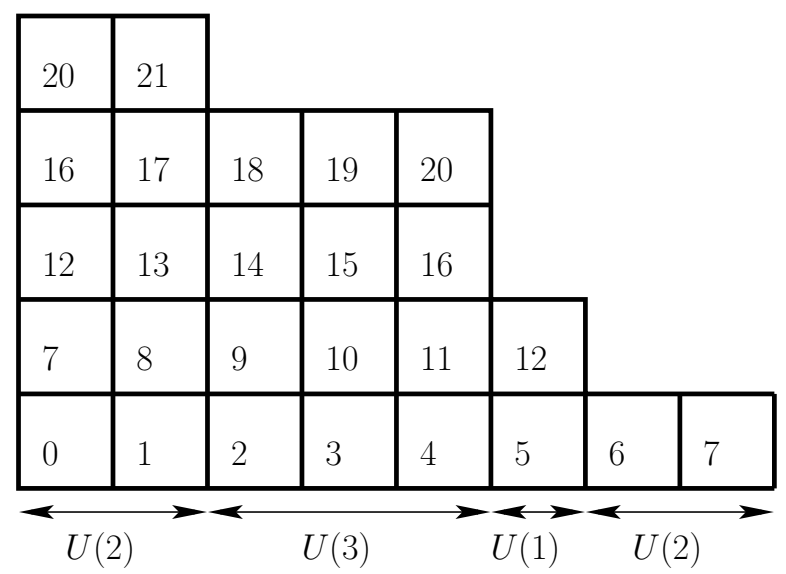

Figure 6: The bottom left box is assigned 0. The assigned integer increases from left to right. As we move up, the first box of each row is assigned the same number as the last box in the row below.

Though the TQFT structure for the Coulomb index is very simple, it is not entirely trivial. We can deduce the Coulomb index of strongly-coupled theories by using dualities, and check that different routes to obtain the index give the same result. For example, using Argyres-Seiberg duality [26]

$$
\mathcal{I}_{E_{6}}=\frac{\mathcal{I}_{S U(3)}^{V}}{\mathcal{I}_{S U(2)}^{V}}=P E\left[\tilde{\mathcal{I}}_{3}\right],
$$

which is the expected result since the Coulomb branch of the $E_{6}$ SCFT is generated by an operator with $E=|r|=3$. Strongly coupled SCFTs are sometimes obtained using S-dualities in more than one way [60] but all the dualities yield the same index, for example

$$
\begin{aligned}
& \mathcal{I}_{E_{6}}=\frac{\mathcal{I}_{S U(3)}^{V}}{\mathcal{I}_{S U(2)}^{V}}=\frac{\mathcal{I}_{S U(4)}^{V}}{\mathcal{I}_{U S p(4)}^{V}}=P E\left[\tilde{\mathcal{I}}_{3}\right] \\
& \mathcal{I}_{E_{7}}=\frac{\mathcal{I}_{S U(4)}^{V}}{\mathcal{I}_{S U(3)}^{V}}=\frac{\mathcal{I}_{U S p(4)}^{V}}{\mathcal{I}_{S U(2)}^{V}}=\frac{\mathcal{I}_{S O(7)}^{V}}{\mathcal{I}_{G_{2}}^{V}}=\frac{\mathcal{I}_{S O(8)}^{V}}{\mathcal{I}_{S O(7)}^{V}}=P E\left[\tilde{\mathcal{I}}_{4}\right] \\
& \mathcal{I}_{E_{8}}=\frac{\mathcal{I}_{S U(6)}^{V}}{\mathcal{I}_{S U(5)}^{V}}=\frac{\mathcal{I}_{U S p(6)}^{V}}{\mathcal{I}_{S O(5)}^{V}}=P E\left[\tilde{\mathcal{I}}_{6}\right] .
\end{aligned}
$$


The index of the $T_{k}$ theory is also obtained easily from the generalized Argyres-Seiberg duality,

$$
\mathcal{I}_{T_{k}}=\frac{\left(\mathcal{I}_{S U(k)}^{V}\right)^{k-2}}{\prod_{j=2}^{k-1} \mathcal{I}_{S U(j)}^{V}}=P E\left[\sum_{j=3}^{k}(j-2) \tilde{\mathcal{I}}_{j}\right] \text {. }
$$

This is again as expected, since the Coulomb branch of the $T_{k}$ theory is spanned by $(j-2)$ operators with $E=|r|=j$, for $j=3, \ldots, k$ (see e.g. [61]).

Extrapolating from these examples let us conjecture the Coulomb index of the theory corresponding to a sphere with three generic punctures. For a general puncture $I$ in the $A_{k-1}$ theory, we associate the set of $k$ numbers $\left\{p_{j}^{(I)}: j=1, \ldots k\right\}$ from the corresponding auxiliary Young diagram. The assignment is illustrated in figure 6. The Coulomb branch index of the theory corresponding to a sphere with three punctures $p^{(1)}, p^{(2)}, p^{(3)}$ is then

$$
\mathcal{I}_{p^{(1)}, p^{(2)}, p^{(3)}}=P E\left[d_{j} \tilde{\mathcal{I}}_{j}\right], \quad d_{j} \equiv \sum_{j=2}^{k}\left(1-2 j+p_{j}^{(1)}+p_{j}^{(2)}+p_{j}^{(3)}\right) .
$$

The dimension $d_{j}$ of the Coulomb branch spanned by operators with $E=|r|=j$ agrees with the dimension of the space of meromorphic $j$-differentials on the Riemann surface having poles of order at most $p_{j}^{(I)}$ at puncture $I[1,43]$.

Let us finally observe that the Coulomb index (8.4) discussed in this section can also be interpreted as the index of $\mathcal{N}=4 \mathrm{SYM}$ in a certain limit of the $\mathcal{N}=4$ superconformal fugacities, such that the index of the $\mathcal{N}=4$ vector multiplet reduces to the index of $\mathcal{N}=2$ vector multiplet. The authors of [62] noticed the appearance of the Macdonald measure in this context.

\section{Discussion}

Let us briefly summarize and discuss our results. We have defined and studied several limits of the $\mathcal{N}=2$ superconformal index. They are characterized by enhanced supersymmetry and depend at most on two superconformal fugacities, out of the possible three. We have given a prescription to calculate these limits for all $A$-series superconformal quivers of class $\mathcal{S}$, even when they lack a Lagrangian description. Thanks to the topological QFT structure of the index, it suffices to find a formula for the elementary three-valent building blocks. For the $S U(2)$ quivers, which do have a Lagrangian description, the building blocks can be written in terms of algebraic objects that admit a natural extrapolation to higher rank, leading to a compelling general conjecture that passes many tests. These objects are the Macdonald polynomials, tailor-made for our purposes as they depend on two fugacities, and for which a beautiful general theory is already available. We expect the generalization of our results to the $D$-series quivers of class $\mathcal{S}$ (and possibly to the $E$-series as well) to be straightforward. 
The TQFT that calculates the index of the $A_{k-1}$ quivers is closely related to twodimensional Yang-Mills theory with gauge group $S U(k)$. An immediate qualitative hint, of course, is that the state-space of the index TQFT is the space of irreducible $S U(k)$ representations. As first discussed in [16], and confirmed here in more generality, there is in fact a precise quantitative correspondence between the limit of the index that we have dubbed the "Schur index", which depends on a single fugacity $q$, and correlators of $q$-deformed $2 d$ Yang-Mills theory [28] in the zero-area limit. In turn, the zero-area limit of $q$-deformed $2 d$ Yang-Mills on the Riemann surface $\mathcal{C}$ can be viewed as an analytic continuation of ChernSimons theory on $\mathcal{C} \times S^{1}[28]$.

Recently, a "refinement" of Chern-Simons theory on three-manifolds admitting a circle action was defined in [31], via the relation with topological string theory and its embedding into M-theory. Taking the three-manifold to be of the form $\mathcal{C} \times S^{1}$, and reducing on the $S^{1}$, one obtains an indirect definition of "refined $q$-deformed Yang-Mills theory" on $\mathcal{C}$, which depends on two parameters $q$ and $t$. (The definition is indirect because unlike the purely $q$-deformed case no Lagrangian description is available for the refined theory.) The refinement essentially amounts to trading Schur polynomials with Macdonald polynomials, and we have found a precise relation between our $(q, t)$ "Macdonald index" and correlators of this $(q, t)$-Yang-Mills theory. It is natural to ask whether this is pointing to a direct connection between topological string theory and the superconformal index. At first sight the geometries involved appear to be quite different, since to obtain the superconformal index we must consider the $(2,0)$ theory on $S^{3} \times S^{1} \times \mathcal{C}$, with appropriate twists induced by the fugacities, while in the setup of $[4,31]$ the relevant geometry is $\left(\mathbb{C} \times S^{1} \times M_{3}\right)_{q, t}$, where one may take $M_{3}=S^{1} \times \mathcal{C}$ (we refer to the cited papers for a proper explanation). Moreover while the index admits a further refinement for a total of three fugacities, it seems difficult to introduce a third parameter in the framework of $[4,31]$ while preserving supersymmetry. Nevertheless, at least for the special case of the Macdonald index, there should be a deeper way to understand the striking similarity of the two results.

An obvious direction for future work is the generalization of our results to the full threeparameter index. The Haar measure together with the index of the $\mathcal{N}=2$ vector multiplet combine to $[19,15]$

$$
\frac{1}{k !} \prod_{i, j=1, i \neq j}^{k} \frac{1}{\Gamma\left(x_{i} / x_{j} ; q, p\right) \Gamma\left(t x_{i} / x_{j} ; q, p\right)}
$$

where $\Gamma(z ; p, q)$ is the elliptic Gamma function

$$
\Gamma(z ; p, q)=\prod_{i, j=0}^{\infty} \frac{1-p^{i+1} q^{j+1} / z}{1-p^{i} q^{j} z} .
$$


A natural speculation is that the functions $f_{p, q, t}^{\lambda}(\mathbf{a})$ that diagonalize the structure constants of the full index should be proportional to elliptic extensions of the Macdonald polynomials, to which they should reduce in the limit $p \rightarrow 0$ (or $q \rightarrow 0$ ). Various proposals for elliptic Macdonald functions have appeared in the mathematical literature, see e.g. [63, 64, 65]. We can in fact formulate a more precise conjecture, motivated by the relation between twodimensional gauge theories and integrable quantum mechanical models of Calogero-Moser (CM) type, see e.g. $[66,67,68,69,70,71]$. The reduction of ordinary $2 d$ Yang-Mills theory to one dimension yields the rational (non-relativistic) CM model [66]. One can consider the trigonometric and elliptic generalizations of the non-relativistic model, as well as their relativistic cousins (the relativistic versions are also known as Ruijsenaars-Schneider (RS) models). The relativistic trigonometric model (trigonometric RS) depends on two parameters $(q, t)$, has Macdonald polynomials as its eigenfunctions, and is closely related to the twodimensional G/G WZW model ${ }^{18}$ or equivalently to Chern-Simons theory on $\mathcal{C} \times S^{1}$. At the summit of this hierarchy is the elliptic relativistic model (elliptic RS), which depends on three parameters, analogous to $(p, q, t)$ of the full index. Our conjecture is then that the symmetric functions relevant for the computation of the full index are the eigenfunctions of the elliptic RS model. Not too much is known about them, see [73] for a review. ${ }^{19}$

Perhaps the most interesting open problem is to give a "microscopic" derivation of the two-dimensional TQFT of the index from the six-dimensional $(2,0)$ theory. A promising shortcut, which exploits the mentioned connection between $2 d$ gauge theories and $1 d$ CalogeroMoser models, is along the following lines. Consider the $(2,0)$ theory on $S^{3} \times S_{(1)}^{1} \times \mathcal{C}_{\mathfrak{g}, s}$. The Riemann surface $\mathcal{C}_{\mathfrak{g}, s}$ can be viewed as a circle, $S_{(2)}^{1}$, times a graph $I_{\mathfrak{g}, s}$ By first reducing the $(2,0)$ theory on $S_{(2)}^{1}$ (note that there is no twist around this circle) one obtains $5 d$ super Yang-Mills on $S^{3} \times S_{(1)}^{1} \times I_{\mathfrak{g}, s}$. We propose that the further reduction of $5 d \mathrm{SYM}$ on $S_{(1)}^{1} \times S^{3}$ (with the fugacity twists) yields the elliptic RS model on the graph $I_{\mathfrak{g}, s}$, with appropriate boundary conditions at the $s$ external punctures and at the internal junctures. In a suitable limit, which corresponds to taking $S_{(1)}^{1}$ to be small, the $4 d$ index becomes the $3 d$ partition function [54, 55, 56], and our proposal reduces to the one of [78] (see also [79]). These authors show how to interpret such $3 d$ partition functions as overlaps of quantum mechanical wave functions. We are suggesting that a similar idea may apply to the $4 d$ index, and that the relevant quantum mechanical model is the elliptic RS model. Work is in progress along these lines.

\footnotetext{
${ }^{18}$ See [70] for a review and [72] for recent relevant work.

${ }^{19}$ Quantum mechanical integrable models have been recently related to the problem of counting vacua of $\mathcal{N}=2$ supersymmetric theories in the $\Omega$-background $[74,75]$. See also $[76,77]$ for connections of elliptic Gamma functions to integrable systems.
} 
Acknowledgments: We would like to thank M. Aganagic, C. Beem, F. van de Bult, T. Dimofte, D. Gaiotto, S. Gukov, D. Jafferis, A. Kirillov jr, J. Maldacena, Y. Nakayama, N. Nekrasov, A. Okounkov, H. Ooguri, E. Rains, B. van Rees, Y. Tachikawa, and E. Witten for very useful discussions. The research of SSR was supported in part by NSF grant PHY0969448 and he would like to thank the Aspen Center for Physics, where part of this work was conducted with the support of the National Science Foundation under Grant No. 1066293. LR thanks the Galileo Galilei Institute for hospitality and the INFN for partial support during the completion of this work. AG would like to thank Tata Institute for Fundamental Research for hospitality during the final stages of this project. This work was supported in part by NSF grant PHY-0969739.

\section{A. Construction of the diagonal expression for the $S U(2)$ HL index}

In this appendix we diagonalize the structure constants of the $S U(2)$ quivers in the $\rho \rightarrow 0$, $\sigma \rightarrow 0$ limit. With hindsight, we have dubbed this limit the Hall-Littlewood (HL) index, since the diagonal functions turn out to be closely related to the Hall-Littlewood polynomials. This is precisely what we show in this appendix.

For $S U(2)$, the SCFT associated to three-punctured sphere is the the free hypermultiplet in the trifundamental representation. In the limit of interest, its index reads

$$
\mathcal{I}(a, b, c)=P E\left[\tau \chi_{1}(a) \chi_{1}(b) \chi_{1}(c)\right]_{a, b, c, \tau}=\frac{1}{\prod_{s_{a}, s_{b}, s_{c}= \pm 1}\left(1-\tau a^{s_{a}} b^{s_{b}} c^{s_{c}}\right)},
$$

where the fugacities $a, b$, and $c$ label the Cartans of the three $S U(2)$ flavor groups. The index of the vector multiplet and the $S U(2)$ Haar measure combine to

$$
\Delta(a) \mathcal{I}^{V}(a, \tau)=\left(1-\tau^{2}\right) \Delta_{\tau^{2}, \tau^{4}}(a)
$$

where $\Delta_{\tau^{2}, \tau^{4}}(a)$ is the Macdonald measure (7.3) with $q=\tau^{2}$ and $t=\tau^{4}$,

$$
\Delta_{\tau^{2}, \tau^{4}}(a)=\frac{1}{2}\left(1-a^{2}\right)\left(1-\frac{1}{a^{2}}\right)\left(1-\tau^{2} a^{2}\right)\left(1-\frac{\tau^{2}}{a^{2}}\right) .
$$

The corresponding Macdonald polynomials $P^{\lambda}\left(a, a^{-1} ; q, t\right)$, normalized to be orthonormal under (A.2), $\operatorname{are}^{20}$

$$
P^{\lambda}\left(a ; \tau^{2}, \tau^{4}\right)=\frac{\tau}{\sqrt{1-\tau^{2}}\left(1-\frac{1}{a^{2}} \tau^{2}\right)\left(1-a^{2} \tau^{2}\right)} \sqrt{\chi_{\lambda}(\tau) \chi_{\lambda+2}(\tau)}\left\{\frac{\chi_{\lambda}(a)}{\chi_{\lambda}(\tau)}-\frac{\chi_{\lambda+2}(a)}{\chi_{\lambda+2}(\tau)}\right\} .
$$

\footnotetext{
${ }^{20}$ This normalization is only used in this appendix. In the rest of the paper Macdonald polynomials are taken to have unit norm with respect to the Macdonald measure.
} 
By choosing $\left\{P^{\lambda}\left(a, a^{-1} ; q, t\right)\right\}$ as a basis, the metric of the TQFT is then trivial, $\eta^{\lambda \mu}=\delta^{\lambda \mu}$. On the other hand, the projection of $\mathcal{I}(a, b, c)$ into the basis functions gives the structure constants $C_{\mu \nu \lambda}$,

$$
\mathcal{I}(a, b, c)=\sum_{\mu, \nu, \lambda=0}^{\infty} C_{\mu \nu \lambda} P^{\mu}\left(a ; \tau^{2}, \tau^{4}\right) P^{\nu}\left(b ; \tau^{2}, \tau^{4}\right) P^{\lambda}\left(c ; \tau^{2}, \tau^{4}\right)
$$

We find that while the structure constants are not diagonal, they take a relatively simple "upper triangular" form. The only non-vanishing coefficients are

$$
C_{\lambda \lambda \lambda} \equiv \Psi_{\lambda}, \quad C_{\lambda \mu \mu}=C_{\mu \lambda \mu}=C_{\mu \mu \lambda} \equiv \Omega_{\lambda} \quad \text { for } \mu<\lambda,(-1)^{\lambda+\mu}=1
$$

where

$$
\begin{aligned}
& \Psi_{\lambda}(\tau)=\frac{\sqrt{1-\tau^{2}}}{\sqrt{\chi_{\lambda+2}(\tau)}}\left(\frac{\tau^{-1}+\tau}{\sqrt{\chi_{\lambda}(\tau)}}-\tau^{\lambda+3} \sqrt{\chi_{\lambda}(\tau)}\right) \\
& \Omega_{\lambda}(\tau)=\sqrt{1-\tau^{2}}\left(\tau^{-1}+\tau\right) \frac{1}{\sqrt{\chi_{\lambda}(\tau) \chi_{\lambda+2}(\tau)}}
\end{aligned}
$$

Associativity is easy to check. It is trivial for most choices of external states, the one interesting case being the four-point function $\mu \mu \nu \nu$ with $\mu<\nu$ and having the same parity (both even or both odd). Equality of the two channels reads

$$
\sum_{\lambda \geq \nu,(-1)^{\lambda+\mu}=1} C_{\mu \mu \lambda} C_{\lambda \nu \nu}=\left[C_{\mu \mu \nu}\right]^{2}
$$

which amounts to (no sum on $\nu$ )

$$
\sum_{\lambda>\nu,(-1)^{\lambda+\mu}=1} \Omega_{\lambda}(\tau)^{2}+\Omega_{\nu}(\tau) \Psi_{\nu}(\tau)=\Omega_{\nu}(\tau)^{2} .
$$

One can verify that this property is satisfied for the particular values of the coefficients given in $(\mathrm{A} .7) .^{21}$

Let us now perform an orthogonal transformation that diagonalizes the structure constants. From (A.6) we see even and odd Macdonald polynomials do not mix with each other and thus can carry our the diagonalization separately for each parity; the discussion below is restricted to the even parity case for definiteness. The Latin letter indices below, $j, \ldots$, run over the integers and correspond to half the value of the Greek indices used above.

We define real symmetric matrices $N_{i}$ as ${ }^{22}$

$$
\left(N_{i}\right)_{j k} \equiv C_{i j k}
$$

\footnotetext{
${ }^{21}$ One needs the identity $\sum_{k=0}^{\infty} \frac{1}{\sinh \alpha(2 k+3) \sinh \alpha(2 k+1)}=\frac{e^{-\alpha}}{2 \sinh ^{2} \alpha \cosh \alpha}$ and induction on $\nu$.

${ }^{22}$ Note that since the metric is trivial, $\eta^{i j}=\delta^{i j}$, the upper or lower position of the indices is immaterial.
} 
Associativity implies that they commute, $\left[N_{i}, N_{j}\right]=0$, so they can be simultaneously diagonalized. Recall that the structure of each matrix $N_{j}$ is

$$
\left(N_{j}\right)_{i k}=\left\{\begin{array}{lr}
i<j, i=k & \Omega_{j} \\
i=k=j & \Psi_{j} \\
i>j, k=j & \Omega_{i} \\
k>j, i=j & \Omega_{k} \\
\text { other } & 0
\end{array}\right.
$$

The non-zero eigenvalues of this matrix are $\Omega_{j}$ with multiplicity $j$, and $\Psi_{j}-\Omega_{j}$ with multiplicity one. The unique eigenvector with eigenvalue $\Psi_{j}-\Omega_{j}$ is

$$
\mathbf{e}_{j+1}=\left(0, \ldots, 0, \Psi_{j}-\Omega_{j}, \Omega_{j+1}, \Omega_{j+2}, \ldots\right),
$$

where there are $j$ zeros in the beginning of the vector. Note that the $\mathbf{e}_{j}$ s are orthogonal to each other,

$$
\mathbf{e}_{j+1} \cdot \mathbf{e}_{k+1}=\left(\Psi_{j}-\Omega_{j}\right) \Omega_{j}+\sum_{i>j}\left[\Omega_{i}\right]^{2}=0
$$

where we took $j>k$ without loss of generality and used the associativity constraint (A.9). Moreover, the vectors $\mathbf{e}_{i}$ turn out to be eigenvectors of all the matrices $N_{j}$,

$$
\begin{array}{lll}
i<j & : & N_{j} \cdot \mathbf{e}_{i+1}=\Omega_{j} \mathbf{e}_{i+1}, \\
i=j & : & N_{j} \cdot \mathbf{e}_{i+1}=\left(\Psi_{j}-\Omega_{j}\right) \mathbf{e}_{i+1}, \\
i>j & : & N_{j} \cdot \mathbf{e}_{i+1}=0 .
\end{array}
$$

This can be shown from the definitions with the help of the associativity constraint (A.9). To complete this set of vectors to a basis we have to add one more vector, orthogonal to all $\mathbf{e}_{j}$,

$$
\mathbf{e}_{0}=\left(\Omega_{1}, \Omega_{2}, \ldots\right) .
$$

This is an eigenvector of all the matrices $N_{j}$ with eigenvalue $\Omega_{j}$. We have thus managed to diagonalize the matrices $N_{i}$. In the diagonal basis $\left\{\mathbf{e}_{j}\right\}$ the matrices are given by (we use hatted indices to represent components in the new basis)

$$
\left(N_{j}\right)_{\hat{i} \hat{k}}=\left\{\begin{array}{lc}
j>\hat{i}, & \Omega_{j} \delta_{\hat{i} \hat{j}} \\
\hat{i}=j, & \left(\Psi_{j}-\Omega_{j}\right) \delta_{\hat{i} \hat{j}} \\
j<\hat{i}, & 0
\end{array}\right.
$$


Finally we perform the orthogonal transformation to the new basis also for the matrix label $j$ of $N_{j}$, and find constants in the new basis read

$$
C_{\hat{j} \hat{i} \hat{k}}=\frac{1}{n_{\hat{j}}} \sum_{l}\left(\mathbf{e}_{\hat{j}}\right)_{l} \cdot\left(N_{l}\right)_{\hat{i} \hat{k}},
$$

where $n_{\hat{j}}$ is the normalization of $\mathbf{e}_{\hat{j}}$,

$$
\begin{array}{ll}
n_{\hat{j}} & =\sqrt{\mathbf{e}_{\hat{j}} \cdot \mathbf{e}_{\hat{j}}}=\tau^{2 \hat{j}} \sqrt{1-\tau^{2}} \quad \text { for } \hat{j}>0, \\
n_{\hat{0}} & =\sqrt{\mathbf{e}_{\hat{0}} \cdot \mathbf{e}_{\hat{0}}}=\sqrt{\left(1-\tau^{2}\right)\left(1+\tau^{2}\right)} .
\end{array}
$$

A little calculation gives

$$
C_{\hat{i} \hat{i} \hat{i}}=n_{\hat{i}}
$$

and zero for the other choices of the indices. So far we have restricted attention to even parity (in terms of the original Greek labels). The case of odd parity works along completely parallel lines.

We can now explicitly compute the functions that diagonalize the structure constants, by contacting the normalized vectors $\mathbf{e}_{\mu} / n_{\mu}$ with the Macdonald polynomials (A.4). A useful identity is $(\lambda>0)$

$$
\sum_{\mu=\lambda,(-1)^{\lambda+\mu}=1}^{\infty} P^{\mu} \Omega_{\mu}=\frac{1+\tau^{2}}{\left(1-\tau^{2} a^{2}\right)\left(1-\tau^{2} / a^{2}\right)} \frac{\chi_{\lambda}(a)}{\chi_{\lambda}(\tau)} .
$$

One finds that the diagonal basis is given by

$$
\begin{aligned}
f^{\lambda}(a, \tau) & =\frac{1}{\sqrt{1-\tau^{2}}} \frac{1}{\left(1-\tau^{2} a^{2}\right)\left(1-\tau^{2} / a^{2}\right)}\left\{\chi_{\lambda}(a)-\tau^{2} \chi_{\lambda-2}(a)\right\} \quad \text { for } \lambda>0, \\
f^{0}(a, \tau) & =\frac{1}{\sqrt{1-\tau^{2}}} \frac{1}{\left(1-\tau^{2} a^{2}\right)\left(1-\tau^{2} / a^{2}\right)} \sqrt{1+\tau^{2}} .
\end{aligned}
$$

It is straightforward to verify that this basis is orthonormal under the measure (A.2). Remarkably, the functions $f^{\lambda}(a, \tau)$ are proportional to the $S U(2)$ Hall-Littlewood polynomials $P_{H L}^{\lambda}\left(a, a^{-1} \mid \tau\right)$, see $(5.11)$, with a $\lambda$-independent proportionality factor $\mathcal{K}(a, \tau)$.

Finally we can write the diagonalized form for the index,

$$
\begin{aligned}
& \mathcal{I}\left(a_{1}, a_{2}, a_{3}\right)= \\
& \quad \frac{1}{1-\tau^{2}} \prod_{i=1}^{3} \frac{1}{\left(1-\tau^{2} a_{i}^{2}\right)\left(1-\tau^{2} / a_{i}^{2}\right)}\left\{\left(1+\tau^{2}\right)^{2}+\sum_{\lambda=1}^{\infty} \tau^{\lambda} \prod_{i=1}^{3}\left(\chi_{\lambda}\left(a_{i}\right)-\tau^{2} \chi_{\lambda-2}\left(a_{i}\right)\right)\right\} .
\end{aligned}
$$

The equality of this expression with (A.1) can be proven directly by elementary means since the sum above is a geometric sum. By noting that

$$
\chi_{\lambda}(\tau)-\tau^{2} \chi_{\lambda-2}(\tau)=\tau^{-\lambda}\left(1+\tau^{2}\right)
$$


and recalling the definition (5.11) of the HL polynomials we can also write

$$
\mathcal{I}\left(a_{1}, a_{2}, a_{3}\right)=\frac{1+\tau^{2}}{1-\tau^{2}} \prod_{i=1}^{3} \frac{1}{\left(1-\tau^{2} a_{i}^{2}\right)\left(1-\tau^{2} / a_{i}^{2}\right)} \sum_{\lambda=0}^{\infty} \frac{1}{P_{\lambda}^{H L}\left(\tau, \tau^{-1} \mid \tau\right)} \prod_{i=1}^{3} P_{\lambda}^{H L}\left(a_{i}, a_{i}^{-1} \mid \tau\right) .
$$

\section{B. Index of short multiplets of $\mathcal{N}=2$ superconformal algebra}

A generic long multiplet $\mathcal{A}_{R, r\left(j_{1}, j_{2}\right)}^{E}$ of the $\mathcal{N}=2$ superconformal algebra is generated by the action of the eight Poincaré supercharges $\mathcal{Q}$ and $\tilde{\mathcal{Q}}$ on a superconformal primary, which by definition is annihilated by all conformal supercharges $\mathcal{S}$. If some combination of the $\mathcal{Q}$ s also annihilates the primary, the corresponding multiplet is shorter and the conformal dimensions of all its members are protected against quantum corrections. The shortening conditions for the $\mathcal{N}=2$ superconformal algebra were studied in [80,81, 82]. We follow the nomenclature of [82], whose classification scheme is summarized in table 4. Let us take a moment to explain the notation. The state $|R, r\rangle_{\left(j_{1}, j_{2}\right)}^{h \cdot w_{0}}$ is the highest weight state with $S U(2)_{R}$ spin $R>0, U(1)_{r}$ charge $r$, which can have either sign, and Lorentz quantum numbers $\left(j_{1}, j_{2}\right)$. The multiplet built on this state is denoted as $\mathcal{X}_{R, r\left(j_{1}, j_{2}\right)}$, where the letter $\mathcal{X}$ characterizes the shortening condition. The left column of table 4 labels the condition. A superscript on the label corresponds to the index $\mathcal{I}=1,2$ of the supercharge that kills the primary: for example $\mathcal{B}_{1}$ refers to $\mathcal{Q}_{1 \alpha}$. Similarly a "bar" on the label refers to the conjugate condition: for example $\overline{\mathcal{B}}_{2}$ corresponds to $\tilde{Q}_{2 \dot{\alpha}}$ annihilating the state; this would result in the short antichiral multiplet $\overline{\mathcal{B}}_{R, r\left(j_{1}, 0\right)}$, obeying $E=2 R-r$. Note that conjugation reverses the signs of $r$, $j_{1}$ and $j_{2}$ in the expression of the conformal dimension.

The superconformal index counts with signs the protected states of the theory, up to equivalence relations that set to zero all sequences of short multiplets that may in principle recombine into long multiplets. The recombination rules for $\mathcal{N}=2$ superconformal algebra are $[82]$

$$
\begin{aligned}
& \mathcal{A}_{R, r\left(j_{1}, j_{2}\right)}^{2 R+r+2 j_{1}+2} \simeq \mathcal{C}_{R, r\left(j_{1}, j_{2}\right)} \oplus \mathcal{C}_{R+\frac{1}{2}, r+\frac{1}{2}\left(j_{1}-\frac{1}{2}, j_{2}\right)}, \\
& \mathcal{A}_{R, r\left(j_{1}, j_{2}\right)}^{2 R-r+2 j_{2}+2} \simeq \overline{\mathcal{C}}_{R, r\left(j_{1}, j_{2}\right)} \oplus \overline{\mathcal{C}}_{R+\frac{1}{2}, r-\frac{1}{2}\left(j_{1}, j_{2}-\frac{1}{2}\right)}, \\
& \mathcal{A}_{R, j_{1}-j_{2}\left(j_{1}, j_{2}\right)}^{2 R+j_{1}+j_{2}+2} \simeq \hat{\mathcal{C}}_{R\left(j_{1}, j_{2}\right)} \oplus \hat{\mathcal{C}}_{R+\frac{1}{2}\left(j_{1}-\frac{1}{2}, j_{2}\right)} \oplus \hat{\mathcal{C}}_{R+\frac{1}{2}\left(j_{1}, j_{2}-\frac{1}{2}\right)} \oplus \hat{\mathcal{C}}_{R+1\left(j_{1}-\frac{1}{2}, j_{2}-\frac{1}{2}\right)} .
\end{aligned}
$$

The $\mathcal{C}, \overline{\mathcal{C}}$ and $\hat{\mathcal{C}}$ multiplets obey certain "semi-shortening" conditions, while $\mathcal{A}$ multiplets are generic long multiplets. A long multiplet whose conformal dimension is exactly at the unitarity threshold can be decomposed into shorter multiplets according to (B.1,B.2,B.3). We can formally regard any multiplet obeying some shortening condition (with the exception of 


\begin{tabular}{|c|c|c|c|c|}
\hline \multicolumn{4}{|c|}{ Shortening Conditions } & \multirow{2}{*}{$\begin{array}{c}\text { Multiplet } \\
\mathcal{B}_{R, r\left(0, j_{2}\right)} \\
\end{array}$} \\
\hline $\mathcal{B}_{1}$ & $\mathcal{Q}_{1 \alpha}|R, r\rangle^{h . w .}=0$ & $j_{1}=0$ & $E=2 R+r$ & \\
\hline$\overline{\mathcal{B}}_{2}$ & $\tilde{\mathcal{Q}}_{2 \dot{\alpha}}|R, r\rangle^{h . w}=0$ & $j_{2}=0$ & $E=2 R-r$ & $\overline{\mathcal{B}}_{R, r\left(j_{1}, 0\right)}$ \\
\hline $\mathcal{E}$ & $\mathcal{B}_{1} \cap \mathcal{B}_{2}$ & $R=0$ & $E=r$ & $\mathcal{E}_{r\left(0, j_{2}\right)}$ \\
\hline$\overline{\mathcal{E}}$ & $\overline{\mathcal{B}}_{1} \cap \overline{\mathcal{B}}_{2}$ & $R=0$ & $E=-r$ & $\overline{\mathcal{E}}_{r\left(j_{1}, 0\right)}$ \\
\hline$\hat{\mathcal{B}}$ & $\mathcal{B}_{1} \cap \bar{B}_{2}$ & $r=0, j_{1}, j_{2}=0$ & $E=2 R$ & $\hat{\mathcal{B}}_{R}$ \\
\hline $\mathcal{C}_{1}$ & $\begin{array}{l}\epsilon^{\alpha \beta} \mathcal{Q}_{1 \beta}|R, r\rangle_{\alpha}^{h . w}=0 \\
\left(\mathcal{Q}_{1}\right)^{2}|R, r\rangle^{h . w}=0 \text { for } j_{1}=0\end{array}$ & & $\begin{array}{l}E=2+2 j_{1}+2 R+r \\
E=2+2 R+r\end{array}$ & $\begin{array}{l}\mathcal{C}_{R, r\left(j_{1}, j_{2}\right)} \\
\mathcal{C}_{R, r\left(0, j_{2}\right)}\end{array}$ \\
\hline$\overline{\mathcal{C}}_{2}$ & $\begin{array}{l}\epsilon^{\dot{\alpha} \dot{\beta}} \tilde{\mathcal{Q}}_{2 \dot{\beta}}|R, r\rangle_{\dot{\alpha}}^{h . w}=0 \\
\left(\tilde{\mathcal{Q}}_{2}\right)^{2}|R, r\rangle^{h . w}=0 \text { for } j_{2}=0\end{array}$ & & $\begin{array}{l}E=2+2 j_{2}+2 R-r \\
E=2+2 R-r\end{array}$ & $\begin{array}{l}\overline{\mathcal{C}}_{R, r\left(j_{1}, j_{2}\right)} \\
\overline{\mathcal{C}}_{R, r\left(j_{1}, 0\right)}\end{array}$ \\
\hline & $\mathcal{C}_{1} \cap \mathcal{C}_{2}$ & $R=0$ & $E=2+2 j_{1}+r$ & $\mathcal{C}_{0, r\left(j_{1}, j_{2}\right)}$ \\
\hline & $\overline{\mathcal{C}}_{1} \cap \overline{\mathcal{C}}_{2}$ & $R=0$ & $E=2+2 j_{2}-r$ & $\overline{\mathcal{C}}_{0, r\left(j_{1}, j_{2}\right)}$ \\
\hline$\hat{\mathcal{C}}$ & $\mathcal{C}_{1} \cap \overline{\mathcal{C}}_{2}$ & $r=j_{2}-j_{1}$ & $E=2+2 R+j_{1}+j_{2}$ & $\hat{\mathcal{C}}_{R\left(j_{1}, j_{2}\right)}$ \\
\hline & $\mathcal{C}_{1} \cap \mathcal{C}_{2} \cap \overline{\mathcal{C}}_{1} \cap \overline{\mathcal{C}}_{2}$ & $R=0, r=j_{2}-j_{1}$ & $E=2+j_{1}+j_{2}$ & $\hat{\mathcal{C}}_{0\left(j_{1}, j_{2}\right)}$ \\
\hline $\mathcal{D}$ & $\mathcal{B}_{1} \cap \overline{\mathcal{C}_{2}}$ & $r=j_{2}+1$ & $E=1+2 R+j_{2}$ & $\mathcal{D}_{R\left(0, j_{2}\right)}$ \\
\hline$\overline{\mathcal{D}}$ & $\overline{\mathcal{B}}_{2} \cap \mathcal{C}_{1}$ & $-r=j_{1}+1$ & $E=1+2 R+j_{1}$ & $\overline{\mathcal{D}}_{R\left(j_{1}, 0\right)}$ \\
\hline & $\mathcal{E} \cap \overline{\mathcal{C}_{2}}$ & $r=j_{2}+1, R=0$ & $E=r=1+j_{2}$ & $\mathcal{D}_{0\left(0, j_{2}\right)}$ \\
\hline & $\overline{\mathcal{E}} \cap \mathcal{C}_{1}$ & $-r=j_{1}+1, R=0$ & $E=-r=1+j_{1}$ & $\overline{\mathcal{D}}_{0\left(j_{1}, 0\right)}$ \\
\hline
\end{tabular}

Table 4: Shortening conditions and short multiplets for the $\mathcal{N}=2$ superconformal algebra.

the $\mathcal{E}(\overline{\mathcal{E}})$ types, and $\overline{\mathcal{D}}_{0\left(j_{1}, 0\right)}\left(\mathcal{D}_{0\left(0, j_{2}\right)}\right)$ types) as a multiplet of type $\mathcal{C}, \overline{\mathcal{C}}$ or $\hat{\mathcal{C}}$ by allowing the spins $j_{1}$ and $j_{2}$, whose natural range is over the non-negative half-integers, to take the value $-1 / 2$ as well. The translation is as follows:

$$
\begin{array}{cc}
\mathcal{C}_{R, r\left(-\frac{1}{2}, j_{2}\right)} \simeq \mathcal{B}_{R+\frac{1}{2}, r+\frac{1}{2}\left(0, j_{2}\right)}, \quad \overline{\mathcal{C}}_{R, r\left(j_{1},-\frac{1}{2}\right)} \simeq \overline{\mathcal{B}}_{R+\frac{1}{2}, r-\frac{1}{2}\left(j_{1}, 0\right)}, \\
\hat{\mathcal{C}}_{R\left(-\frac{1}{2}, j_{2}\right)} \simeq \mathcal{D}_{R+\frac{1}{2}\left(0, j_{2}\right)}, \quad \hat{\mathcal{C}}_{R\left(j_{1},-\frac{1}{2}\right)} \simeq \overline{\mathcal{D}}_{R+\frac{1}{2}\left(j_{1}, 0\right)}, \\
\hat{\mathcal{C}}_{R\left(-\frac{1}{2},-\frac{1}{2}\right)} \simeq \mathcal{D}_{R+\frac{1}{2}\left(0,-\frac{1}{2}\right)} \simeq \overline{\mathcal{D}}_{R+\frac{1}{2}\left(-\frac{1}{2}, 0\right)} \simeq \hat{\mathcal{B}}_{R+1} .
\end{array}
$$

Note how these rules flip statistics: a multiplet with bosonic primary $\left(j_{1}+j_{2}\right.$ integer $)$ is turned into a multiplet with fermionic primary $\left(j_{1}+j_{2}\right.$ half-odd), and vice versa. With these 
conventions, the rules (B.1, B.2, B.3) are the most general recombination rules. The $\mathcal{E}$ and $\overline{\mathcal{E}}$ multiplets never recombine.

The index of the $\mathcal{C}$ and $\mathcal{E}$ type multiplets vanishes identically (the choice of supercharge with respect to which the index is computed, $\mathcal{Q}=\widetilde{\mathcal{Q}}_{1-}$, breaks the symmetry between $\mathcal{C}(\mathcal{E})$ and $\overline{\mathcal{C}}(\overline{\mathcal{E}})$ multiplets). The index of all remaining short multiplets can be specified by listing the index of $\overline{\mathcal{C}}, \hat{\mathcal{C}}, \overline{\mathcal{E}}, \mathcal{D}_{0\left(0, j_{2}\right)}$, and $\overline{\mathcal{D}}_{0\left(j_{1}, 0\right)}$ multiplets,

$$
\begin{aligned}
& \mathcal{I}_{\overline{\mathcal{C}}_{R, r\left(j_{1}, j_{2}\right)}}=-(-1)^{2\left(j_{1}+j_{2}\right)} \tau^{2+2 R+2 j_{2}} \sigma^{j_{2}-r} \rho^{j_{2}-r} \frac{(1-\sigma \rho)(\tau-\sigma)(\tau-\rho)}{(1-\sigma \tau)(1-\rho \tau)} \chi_{2 j_{1}}\left(\sqrt{\frac{\sigma}{\rho}}\right), \\
& \mathcal{I}_{\hat{\mathcal{C}}_{R\left(j_{1}, j_{2}\right)}}=(-1)^{2\left(j_{1}+j_{2}\right)} \frac{\tau^{3+2 R+2 j_{2}} \sigma^{j_{1}+\frac{1}{2}} \rho^{j_{1}+\frac{1}{2}}(1-\sigma \rho)}{(1-\sigma \tau)(1-\rho \tau)}\left(\chi_{2 j_{1}+1}\left(\sqrt{\frac{\sigma}{\rho}}\right)-\frac{\sqrt{\sigma \rho}}{\tau} \chi_{2 j_{1}}\left(\sqrt{\frac{\sigma}{\rho}}\right)\right), \\
& \mathcal{I}_{\overline{\mathcal{E}}_{r\left(j_{1}, 0\right)}}=(-1)^{2 j_{1}} \sigma^{-r-1} \rho^{-r-1} \frac{(\tau-\sigma)(\tau-\rho)}{(1-\sigma \tau)(1-\rho \tau)} \chi_{2 j_{1}}\left(\sqrt{\frac{\sigma}{\rho}}\right), \\
& \mathcal{I}_{\overline{\mathcal{D}}_{0\left(j_{1}, 0\right)}}= \frac{(-1)^{2 j_{1}}(\sigma \rho)^{j_{1}+1}}{(1-\sigma \tau)(1-\rho \tau)} \times \\
& \quad\left(\left(1+\tau^{2}\right) \chi_{2 j_{1}}\left(\sqrt{\frac{\sigma}{\rho}}\right)-\frac{\tau}{\sqrt{\sigma \rho}} \chi_{2 j_{1}+1}\left(\sqrt{\frac{\sigma}{\rho}}\right)-\tau \sqrt{\sigma \rho} \chi_{2 j_{1}-1}\left(\sqrt{\frac{\sigma}{\rho}}\right)\right), \\
& \mathcal{I}_{\mathcal{D}_{0\left(0, j_{2}\right)}=} \frac{(-1)^{2 j_{2}+1} \tau^{2 j_{2}+2}}{(1-\sigma \tau)(1-\rho \tau)}(1-\sigma \rho) .
\end{aligned}
$$

where the Schur polynomial $\chi_{2 j}\left(\sqrt{\frac{\sigma}{\rho}}\right)$ gives the character of the spin $j$ representation of $S U(2)$.

Let us evaluate the interesting limits of the index studied in this paper on individual multiplets.

\section{Macdonald index}

This index is obtained from the general index in the limit $\sigma \rightarrow 0$. The index of the short multiplets in this limit is given by

$$
\begin{aligned}
\mathcal{I}_{\overline{\mathcal{C}}_{R, r\left(j_{1}, j_{2}\right)}} & =0 \\
\mathcal{I}_{\hat{\mathcal{C}}_{R\left(j_{1}, j_{2}\right)}} & =(-1)^{2\left(j_{1}+j_{2}\right)} \frac{\tau^{3+2 R+2 j_{2}} \rho^{2 j_{1}+1}}{(1-\rho \tau)} \\
\mathcal{I}_{\overline{\mathcal{E}}_{r\left(j_{1}, 0\right)}} & =0, \\
\mathcal{I}_{\overline{\mathcal{D}}_{0\left(j_{1}, 0\right)}} & =(-1)^{2 j_{1}+1} \frac{\tau \rho^{2 j_{1}+1}}{(1-\rho \tau)}, \quad \mathcal{I}_{\mathcal{D}_{0\left(0, j_{2}\right)}}=(-1)^{2 j_{2}+1} \frac{\tau^{2 j_{2}+2}}{(1-\rho \tau)} .
\end{aligned}
$$

While taking the limit of the $\overline{\mathcal{C}}$ and $\overline{\mathcal{E}}$ multiplet index we have used $j_{2}-j_{1}>r$ and $-r>j_{1}+1$ respectively. The first inequality follows from the bound $\delta_{1-} \geq 0$ along with $\tilde{\delta}_{1-}=0$ and the second one can be obtained by evaluating $\delta_{1-} \geq 0$ on the first descendant of the primary of the $\overline{\mathcal{E}}$ multiplet. 


\section{Hall-Littlewood index}

This index is obtained from the Macdonald index by further taking the limit $\rho \rightarrow 0$. The index of the short multiplets is

$$
\begin{aligned}
\mathcal{I}_{\overline{\mathcal{C}}_{R, r\left(j_{1}, j_{2}\right)}} & =0 \\
\mathcal{I}_{\hat{\mathcal{C}}_{R\left(j_{1}, j_{2}\right)}} & =-(-1)^{2 j_{2}} \tau^{3+2 R+2 j_{2}} \delta_{j_{1},-\frac{1}{2}}, \\
\mathcal{I}_{\overline{\mathcal{E}}_{r\left(j_{1}, 0\right)}} & =0, \\
\mathcal{I}_{\overline{\mathcal{D}}_{0\left(j_{1}, 0\right)}} & =0, \quad \mathcal{I}_{\mathcal{D}_{0\left(0, j_{2}\right)}}=(-1)^{2 j_{2}+1} \tau^{2 j_{2}+2} .
\end{aligned}
$$

\section{Schur Index}

We take the limit $\tau \rightarrow \rho$. In this limit, the index becomes independent of $\sigma$ and the short multiplets give

$$
\begin{aligned}
\mathcal{I}_{\overline{\mathcal{C}}_{R, r\left(j_{1}, j_{2}\right)}} & =0 \\
\mathcal{I}_{\hat{\mathcal{C}}_{R\left(j_{1}, j_{2}\right)}} & =(-1)^{2\left(j_{1}+j_{2}\right)} \frac{\tau^{4+2\left(R+j_{1}+j_{2}\right)}}{\left(1-\tau^{2}\right)} \\
\mathcal{I}_{\overline{\mathcal{E}}_{r\left(j_{1}, 0\right)}} & =0, \\
\mathcal{I}_{\overline{\mathcal{D}}_{0\left(j_{1}, 0\right)}} & =(-1)^{2 j_{1}+1} \frac{\tau^{2 j_{1}+2}}{\left(1-\tau^{2}\right)}, \quad \mathcal{I}_{\mathcal{D}_{0\left(0, j_{2}\right)}}=(-1)^{2 j_{2}+1} \frac{\tau^{2 j_{2}+2}}{\left(1-\tau^{2}\right)} .
\end{aligned}
$$

\section{Coulomb Index}

Finally we take $\tau \rightarrow 0$. In this limit only the $\overline{\mathcal{E}}$ multiplet have a non-vanishing index

$$
\begin{aligned}
\mathcal{I}_{\overline{\mathcal{C}}_{R, r\left(j_{1}, j_{2}\right)}} & =0, \\
\mathcal{I}_{\hat{\mathcal{C}}_{R\left(j_{1}, j_{2}\right)}} & =0, \\
\mathcal{I}_{\overline{\mathcal{E}}_{r\left(j_{1}, 0\right)}} & =(-1)^{2 j_{1}}(\sigma \rho)^{-r} \chi_{2 j_{1}}\left(\sqrt{\frac{\sigma}{\rho}}\right), \\
\mathcal{I}_{\overline{\mathcal{D}}_{0\left(j_{1}, 0\right)}} & =(-1)^{2 j_{1}}(\sigma \rho)^{j_{1}+1} \chi_{2 j_{1}}\left(\sqrt{\frac{\sigma}{\rho}}\right), \quad \mathcal{I}_{\mathcal{D}_{0\left(0, j_{2}\right)}}=0 .
\end{aligned}
$$

The $\mathcal{N}=2$ vector multiplet is the direct sum of $\mathcal{D}_{0(0,0)}$ and $\overline{\mathcal{D}}_{0(0,0)}$, indeed $(4.17)$ is simply $\overline{\mathcal{D}}_{0(0,0)} \cdot{ }^{23}$ In a Lagrangian theory, the only possible $\overline{\mathcal{D}}$ multiplets have $j_{1}=0$, and are obtained from the $\overline{\mathcal{D}}_{0(0,0)}$ half of the $\mathcal{N}=2$ vector multiplet. In the less restrictive limit of $\sigma, \tau \rightarrow 0$ and $\rho \rightarrow \infty$ the index of some of the short multiplets could potentially diverge. However, for Lagrangian theories the only contributing multiplets are $\overline{\mathcal{E}}_{r(0,0)}$ multiplets arising from tensor products of the $\overline{\mathcal{D}}_{0(0,0)}$ from the vector multiplet, whose index is finite.

\footnotetext{
${ }^{23}$ Note that in this limit $\mathcal{I}_{\overline{\mathcal{E}}_{-1(0,0)}}=\mathcal{I}_{\overline{\mathcal{D}}_{0(0,0)}}$. This is also true in the less restrictive Coulomb limit (4.18).
} 


\section{Large $k$ limit of the genus $\mathfrak{g}$ HL index}

In this appendix we give some details about the large $k$ limit of the HL index for $S U(k)$ quivers corresponding to genus $\mathfrak{g}$ surface with no punctures. For finite $k$, the index is given by $(5.36)$,

$$
\mathcal{I}_{\mathfrak{g}}^{(k)}=\frac{\left(\prod_{j=2}^{k}\left(1-\tau^{2 j}\right)\right)^{2 \mathfrak{g}-2}}{\left(1-\tau^{2}\right)^{(k-1)(\mathfrak{g}-1)}} \sum_{\lambda} \frac{1}{P_{\lambda}^{H L}\left(\tau^{k-1}, \tau^{k-3}, \ldots, \tau^{1-k} \mid \tau\right)^{2 \mathfrak{g}-2}},
$$

The denominator in the sum above is explicitly given by [42],

$$
P_{\lambda}^{H L}\left(\tau^{k-1}, \tau^{k-3}, \ldots, \tau^{1-k} \mid \tau\right)=\mathcal{N}_{\lambda}(\tau) \tau^{\sum_{i=1}^{k-1}(2 i-k-1) \lambda_{i}} \prod_{i=1}^{k} \frac{1-\tau^{2 i}}{1-\tau^{2}},
$$

where $\mathcal{N}_{\lambda}(\tau)$ is given in (5.19),

$$
\mathcal{N}_{\lambda_{1}, \ldots \lambda_{k}}^{-2}(\tau)=\prod_{i=0}^{\infty} \prod_{j=1}^{m(i)}\left(\frac{1-\tau^{2 j}}{1-\tau^{2}}\right)
$$

Here $m(i)$ is the number of rows in the Young diagram $\lambda=\left(\lambda_{1}, \ldots, \lambda_{k}\right)$ of length $i$. We need to evaluate

$$
\begin{aligned}
\mathcal{I}_{\mathfrak{g}}^{(k)} & =\left(1-\tau^{2}\right)^{(k-1)(\mathfrak{g}-1)} \sum_{\lambda_{1} \geq \lambda_{2} \geq \cdots \geq \lambda_{k-1}} \mathcal{N}_{\lambda_{1}, \ldots, \lambda_{k-1}, 0}^{2-2 \mathfrak{g}} \tau^{-(2 \mathfrak{g}-2) \sum_{i=1}^{k-1}(2 i-k-1) \lambda_{i}}= \\
& =\left(1-\tau^{2}\right)^{(k-1)(\mathfrak{g}-1)} \sum_{\eta_{1}, \eta_{2}, \ldots, \eta_{k-1}=0}^{\infty} \mathcal{N}_{\eta_{1}, \ldots, \eta_{k-1}}^{2-2 \mathfrak{g}} \tau^{(2 \mathfrak{g}-2) \sum_{i=1}^{k-1}(k-i) i \eta_{i}},
\end{aligned}
$$

where $\lambda_{i}=\sum_{j=1}^{k-i} \eta_{k-j}$. In the large $k$ limit terms with non-zero $\eta_{i}$ vanish since we always assume $|\tau| \ll 1$. Thus, the only contribution to the sum at leading order for large $k$ is from the term with all $\eta_{i}=0$,

$$
\begin{aligned}
\mathcal{I}_{\mathfrak{g}}^{(k \rightarrow \infty)} & =\lim _{k \rightarrow \infty}\left(1-\tau^{2}\right)^{(k-1)(\mathfrak{g}-1)} \mathcal{N}_{\lambda_{1}=0, \ldots, \lambda_{k-1}=0,0}^{2-2 \mathfrak{g}}= \\
& =\prod_{j=2}^{\infty}\left(1-\tau^{2 j}\right)^{\mathfrak{g}-1}=P E\left[-(\mathfrak{g}-1) \frac{\tau^{4}}{1-\tau^{2}}\right] .
\end{aligned}
$$

The same logic applies also to the large $k$ limit of the $T_{k}$ theories: the singlet is the only term contributing to the index at leading order. 


\begin{tabular}{|l|l|l|l|}
\hline 1 & $\tau$ & $33 \tau^{2}$ & $144 \tau^{3}$ \\
$873 \tau^{4}$ & $4169 \tau^{5}$ & $19486 \tau^{6}$ & $80693 \tau^{7}$ \\
$319237 \tau^{8}$ & $1165632 \tau^{9}$ & $4024927 \tau^{10}$ & $13054735 \tau^{11}$ \\
$40137244 \tau^{12}$ & $116876141 \tau^{13}$ & $323853313 \tau^{14}$ & $854555364 \tau^{15}$ \\
$2153519932 \tau^{16}$ & $5188980328 \tau^{17}$ & $11978372385 \tau^{18}$ & $26521974729 \tau^{19}$ \\
$56409853881 \tau^{20}$ & $115373040784 \tau^{21}$ & $227178289971 \tau^{22}$ & $431064583235 \tau^{23}$ \\
$788945072797 \tau^{24}$ & $1393870863434 \tau^{25}$ & $2379094134408 \tau^{26}$ & $3925581861006 \tau^{27}$ \\
$6265884973841 \tau^{28}$ & $9680331918067 \tau^{29}$ & $14483072164070 \tau^{30}$ & $20994033528147 \tau^{31}$ \\
$29497595795349 \tau^{32}$ & $40188148151858 \tau^{33}$ & $53110900086737 \tau^{34}$ & $68104402838959 \tau^{35}$ \\
$84760383950971 \tau^{36}$ & $102408879854636 \tau^{37}$ & $120143187852325 \tau^{38}$ & $136883008184825 \tau^{39}$ \\
$151478220483799 \tau^{40}$ & $162834262989902 \tau^{41}$ & $170047651342244 \tau^{42}$ & $172521386089030 \tau^{43}$ \\
\hline
\end{tabular}

Table 5: The coefficients of $\mathcal{P}_{86}(\tau)$. The coefficient of $\tau^{86-k}$ is equal to the coefficient of $\tau^{k}$.

\section{The unrefined HL index of $T_{4}$}

Using the conjecture of section 5.4 we can write an explicit expression for the unrefined index of the $T_{4}$ theory. We find

$$
\mathcal{I}_{T_{4}}=\frac{\left(1-\tau^{4}\right)\left(1-\tau^{6}\right)\left(1-\tau^{8}\right)}{\left(1-\tau^{2}\right)^{42}} \sum_{\lambda_{1} \geq \lambda_{2} \geq \lambda_{3} \geq 0} \frac{\left(P_{\lambda_{1}, \lambda_{2}, \lambda_{3}}^{H L}(1,1,1,1 \mid \beta)\right)^{3}}{P_{\lambda_{1}, \lambda_{2}, \lambda_{3}}^{H L}\left(\tau^{3}, \tau, \tau^{-1}, \tau^{-3} \mid \beta\right)}
$$

The sum over the representation can be explicitly evaluated to give

$$
\mathcal{I}_{T_{4}}=\frac{1-\tau}{\left(1-\tau^{2}\right)^{13}\left(1-\tau^{3}\right)^{17}\left(1-\tau^{4}\right)^{13}} \mathcal{P}_{86}(\tau)
$$

where $\mathcal{P}_{86}(\tau)$ is a polyndromic polynomial of degree 86 in $\tau$ with coefficients given in table 5 . The degree of the singularity when $\tau \rightarrow 1$ has a physical meaning: since the Hall-Littlewood index computes the Hilbert series of the Higgs branch this is the complex dimension of the Higgs branch. For $T_{4}$ the HL index predicts the dimension to be 42 , in agreement with $[47,61]$. 


\section{E. Proof of the $S U(2)$ Schur index identity}

In this appendix we prove the basic $S U(2)$ Schur index identity (6.11),

$$
\frac{P E\left[\frac{q^{1 / 2}}{1-q}\left(a_{1}+\frac{1}{a_{1}}\right)\left(a_{2}+\frac{1}{a_{2}}\right)\left(a_{3}+\frac{1}{a_{3}}\right)\right]_{a_{i}, q}}{(q ; q)^{3}\left(q^{2} ; q\right) \prod_{i=1}^{3} P E\left[\frac{q}{1-q}\left(a_{i}^{2}+a_{i}^{-2}+2\right)\right]_{a_{i}, q}}=\sum_{\lambda=0}^{\infty} \frac{\prod_{i=1}^{3} \chi_{\lambda}\left(a_{i}, a_{i}^{-1}\right)}{\chi_{\lambda}\left(q^{\frac{1}{2}}, q^{-\frac{1}{2}}\right)} .
$$

The strategy is to study the analytic properties of this expression and show that the left- and right-handed sides have the same poles and residues. Let us first define

$$
x=\frac{a_{1}}{a_{2} a_{3}}, \quad y=\frac{a_{2}}{a_{1} a_{3}}, \quad z=\frac{a_{3}}{a_{2} a_{1}}, \quad u=a_{1} a_{2} a_{3}, \quad x y z u=1,
$$

where $a_{i}$ are $S U(2)$ fugacities. We also define

$$
(a) \equiv(a ; q)_{\infty} \equiv \prod_{i=0}^{\infty}\left(1-a q^{i}\right)
$$

We will use square brackets [ ] to denote ordinary brackets (that delimit expressions). Then, using

$$
\sum_{n=1}^{\infty} \frac{x^{n}}{n}=-\log (1-x)
$$

the LHS of (E.1) is given by

$$
L H S=\frac{[1-q](q)^{2}(q x y)(q x z)(q x u)(q y z)(q y u)(q z u)}{\left(q^{1 / 2} x\right)\left(q^{1 / 2} / x\right)\left(q^{1 / 2} y\right)\left(q^{1 / 2} / y\right)\left(q^{1 / 2} z\right)\left(q^{1 / 2} / z\right)\left(q^{1 / 2} u\right)\left(q^{1 / 2} / u\right)} .
$$

Let us study the analytic properties of this expression as a function of $x$ (the expression is symmetric in $x, y, z, u$ ). We have poles whenever $x=q^{1 / 2-l}$ with integer $l$ (positive, zero or negative). At $x \rightarrow 0, \infty$ we have accumulation of poles. Let us for concreteness compute the residue with positive $l$

$$
\operatorname{Res}_{L H S}=\frac{[1-q](q)^{2}\left(q^{3 / 2-l} y\right)\left(q^{3 / 2-l} z\right)(q /(y z))(q y z)\left(q^{1 / 2+l} / z\right)\left(q^{1 / 2+l} / y\right)}{\left(q^{1-l}\right)^{\prime}\left(q^{l}\right)\left(q^{1 / 2} y\right)\left(q^{1 / 2} / y\right)\left(q^{1 / 2} z\right)\left(q^{1 / 2} / z\right)\left(q^{l} /(y z)\right)\left(q^{1-l} y z\right)} .
$$

Here $\left(q^{1-l}\right)^{\prime}$ is $\left(q^{1 / 2} x\right)$ evaluated at $x=q^{1 / 2-l}$ with the vanishing factor removed. Now we have

$$
\frac{\left(q^{1 / 2-l+1} y\right)\left(q^{1 / 2+l} / y\right)}{\left(q^{1 / 2} y\right)\left(q^{1 / 2} / y\right)}=\frac{[-y]^{l}}{q^{l^{2} / 2}} \frac{1}{1-q^{1 / 2-l} y}
$$

From here we get

$$
\operatorname{Res}_{L H S}=\frac{[1-q](q)^{2}[y z]^{l} \prod_{i=0}^{l-2}\left(1-q^{1+i} /(y z)\right)}{\left(q^{1-l}\right)^{\prime}\left(q^{l}\right) q^{l^{2}}\left[1-q^{1 / 2-l} y\right]\left[1-q^{1 / 2-l} z\right] \prod_{i=0}^{l-1}\left(1-q^{-i} y z\right)}=\frac{q^{-1 / 2}-q^{1 / 2}}{A}
$$


where

$$
A=x-\frac{1}{x}+y-\frac{1}{y}+z-\frac{1}{z}+u-\frac{1}{u} .
$$

Let us now look on the RHS of (E.1), which can be written as

$$
R H S=\frac{q^{-1 / 2}-q^{1 / 2}}{A} \sum_{n=1}^{\infty} \frac{q^{n / 2}}{1-q^{n}}\left(x^{n}-\frac{1}{x^{n}}+y^{n}-\frac{1}{y^{n}}+z^{n}-\frac{1}{z^{n}}+u^{n}-\frac{1}{u^{n}}\right) .
$$

We again want to compute residues in $x$. To see the poles we write

$$
\sum_{i=1}^{\infty} \frac{q^{n / 2}}{1-q^{n}} x^{n}=\sum_{i=0}^{\infty} \sum_{n=1}^{\infty} q^{n(1 / 2+i)} x^{n}=\sum_{i=0}^{\infty} \frac{q^{1 / 2+i} x}{1-q^{1 / 2+i} x} .
$$

Thus again the poles are at $x=q^{1 / 2-l}$ for any integer $l$ (we have also same expression as (E.11) with $x \rightarrow 1 / x)$. The residue here is easily computed to give

$$
\operatorname{Res}_{R H S}=\frac{q^{-1 / 2}-q^{1 / 2}}{A}
$$

All in all, the LHS and RHS have the same poles and residues. 


\section{References}

[1] D. Gaiotto, N=2 dualities, arXiv:0904.2715.

[2] D. Gaiotto, G. W. Moore, and A. Neitzke, Wall-crossing, Hitchin Systems, and the WKB Approximation, 0907.3987.

[3] S. Cecotti and C. Vafa, Classification of complete $N=2$ supersymmetric theories in 4 dimensions, 1103.5832 .

[4] S. Cecotti, A. Neitzke, and C. Vafa, R-Twisting and 4d/2d Correspondences, 1006.3435.

[5] M. Alim, S. Cecotti, C. Cordova, S. Espahbodi, A. Rastogi, et al., BPS Quivers and Spectra of Complete N=2 Quantum Field Theories, 1109.4941.

[6] M. T. Anderson, C. Beem, N. Bobev, and L. Rastelli, Holographic Uniformization, 1109.3724.

[7] L. F. Alday, D. Gaiotto, and Y. Tachikawa, Liouville Correlation Functions from Four-dimensional Gauge Theories, Lett. Math. Phys. 91 (2010) 167-197, [0906.3219].

[8] N. Wyllard, $A_{N-1}$ conformal Toda field theory correlation functions from conformal $N=2$ SU(N) quiver gauge theories, JHEP 11 (2009) 002, [0907.2189].

[9] A. Mironov and A. Morozov, On AGT relation in the case of U(3), Nucl.Phys. B825 (2010) $1-37,[0908.2569]$.

[10] N. A. Nekrasov, Seiberg-Witten Prepotential From Instanton Counting, Adv. Theor. Math. Phys. 7 (2004) 831-864, [hep-th/0206161].

[11] V. Pestun, Localization of gauge theory on a four-sphere and supersymmetric Wilson loops, 0712.2824.

[12] J. Kinney, J. M. Maldacena, S. Minwalla, and S. Raju, An index for 4 dimensional super conformal theories, Commun. Math. Phys. 275 (2007) 209-254, [hep-th/0510251].

[13] C. Romelsberger, Counting chiral primaries in $N=1, d=4$ superconformal field theories, Nucl. Phys. B747 (2006) 329-353, [hep-th/0510060].

[14] A. Gadde, E. Pomoni, L. Rastelli, and S. S. Razamat, S-duality and 2d Topological QFT, JHEP 03 (2010) 032, [0910.2225].

[15] A. Gadde, L. Rastelli, S. S. Razamat, and W. Yan, The Superconformal Index of the $E_{6} S C F T$, JHEP 08 (2010) 107, [1003.4244].

[16] A. Gadde, L. Rastelli, S. S. Razamat, and W. Yan, The 4d Superconformal Index from q-deformed 2d Yang-Mills, Phys. Rev. Lett. 106 (2011) 241602, [1104.3850].

[17] C. Romelsberger, Calculating the Superconformal Index and Seiberg Duality, 0707.3702.

[18] G. Festuccia and N. Seiberg, Rigid Supersymmetric Theories in Curved Superspace, JHEP 1106 (2011) 114, [1105.0689]. 
[19] F. A. Dolan and H. Osborn, Applications of the Superconformal Index for Protected Operators and q-Hypergeometric Identities to N=1 Dual Theories, Nucl. Phys. B818 (2009) 137-178, [arXiv:0801.4947].

[20] V. P. Spiridonov and G. S. Vartanov, Superconformal indices for $\mathcal{N}=1$ theories with multiple duals, Nucl. Phys. B824 (2010) 192-216, [0811.1909].

[21] V. P. Spiridonov and G. S. Vartanov, Elliptic hypergeometry of supersymmetric dualities, 0910.5944.

[22] V. Spiridonov and G. Vartanov, Supersymmetric dualities beyond the conformal window, Phys.Rev.Lett. 105 (2010) 061603, [1003.6109].

[23] A. Gadde, L. Rastelli, S. S. Razamat, and W. Yan, On the Superconformal Index of N=1 IR Fixed Points: A Holographic Check, JHEP 1103 (2011) 041, [1011.5278].

[24] G. Vartanov, On the ISS model of dynamical SUSY breaking, Phys.Lett. B696 (2011) 288-290, [1009.2153].

[25] V. Spiridonov and G. Vartanov, Elliptic hypergeometry of supersymmetric dualities II. Orthogonal groups, knots, and vortices, 1107.5788.

[26] P. C. Argyres and N. Seiberg, S-duality in n=2 supersymmetric gauge theories, JHEP 0712 (2007) 088.

[27] J. A. Minahan and D. Nemeschansky, An $N=2$ superconformal fixed point with E(6) global symmetry, Nucl. Phys. B482 (1996) 142-152, [hep-th/9608047].

[28] M. Aganagic, H. Ooguri, N. Saulina, and C. Vafa, Black holes, q-deformed 2d Yang-Mills, and non-perturbative topological strings, Nucl.Phys. B715 (2005) 304-348, [hep-th/0411280].

[29] E. Buffenoir and P. Roche, Two-dimensional lattice gauge theory based on a quantum group, Commun. Math. Phys. 170 (1995) 669-698, [hep-th/9405126].

[30] C. Klimcik, The formulae of Kontsevich and Verlinde from the perspective of the Drinfeld double, Commun. Math. Phys. 217 (2001) 203-228, [hep-th/9911239].

[31] M. Aganagic and S. Shakirov, Knot Homology from Refined Chern-Simons Theory, 1105.5117.

[32] S. Benvenuti, A. Hanany, and N. Mekareeya, The Hilbert Series of the One Instanton Moduli Space, JHEP 06 (2010) 100, [1005.3026].

[33] A. Hanany and N. Mekareeya, Tri-vertices and SU(2)'s, JHEP 1102 (2011) 069, [1012.2119].

[34] V. Spiridonov, Elliptic hypergeometric functions, arXiv:0704.3099.

[35] A. Gadde, E. Pomoni, and L. Rastelli, The Veneziano Limit of N=2 Superconformal QCD: Towards the String Dual of $N=2 S U\left(N_{c}\right) S Y M$ with $N_{f}=2 N_{c}, 0912.4918$.

[36] E. Witten, On quantum gauge theories in two-dimensions, Commun.Math.Phys. 141 (1991) 153-209. 
[37] E. Witten, Two-dimensional gauge theories revisited, J. Geom. Phys. 9 (1992) 303-368, [hep-th/9204083].

[38] S. Cordes, G. W. Moore, and S. Ramgoolam, Lectures on 2-d Yang-Mills theory, equivariant cohomology and topological field theories, Nucl. Phys. Proc. Suppl. 41 (1995) 184-244, [hep-th/9411210].

[39] J. Gray, A. Hanany, Y.-H. He, V. Jejjala, and N. Mekareeya, SQCD: A Geometric Apercu, JHEP 0805 (2008) 099, [0803.4257].

[40] A. Hanany and N. Mekareeya, Counting Gauge Invariant Operators in SQCD with Classical Gauge Groups, JHEP 0810 (2008) 012, [0805.3728].

[41] J. A. Minahan and D. Nemeschansky, Superconformal fixed points with E(n) global symmetry, Nucl. Phys. B489 (1997) 24-46, [hep-th/9610076].

[42] I. G. Macdonald, Symmetric functions and hall polynomials, Oxford University Press (1995).

[43] O. Chacaltana and J. Distler, Tinkertoys for Gaiotto Duality, JHEP 1011 (2010) 099, [1008.5203].

[44] G. W. Moore and Y. Tachikawa, On 2d TQFTs whose values are holomorphic symplectic varieties, 1106.5698.

[45] D. Gaiotto, G. W. Moore, and Y. Tachikawa, On $6 d N=(2,0)$ theory compactified on a Riemann surface with finite area, 1110.2657.

[46] D. Gaiotto, A. Neitzke, and Y. Tachikawa, Argyres-Seiberg duality and the Higgs branch, Commun. Math. Phys. 294 (2010) 389-410, [0810.4541].

[47] D. Gaiotto and J. Maldacena, The gravity duals of N=2 superconformal field theories, arXiv:0904.4466.

[48] Y. Nakayama, Index for orbifold quiver gauge theories, Phys. Lett. B636 (2006) 132-136, [hep-th/0512280].

[49] Y. Nakayama, Index for supergravity on $\operatorname{AdS}(5) x T^{* * 1}, 1$ and conifold gauge theory, Nucl.Phys. B755 (2006) 295-312, [hep-th/0602284].

[50] H. Awata and Y. Yamada, Five-dimensional AGT Conjecture and the Deformed Virasoro Algebra, JHEP 01 (2010) 125, [0910.4431].

[51] R. Schiappa and N. Wyllard, An Ar threesome: Matrix models, 2d CFTs and $4 d N=2$ gauge theories, 0911.5337.

[52] A. Mironov, A. Morozov, S. Shakirov, and A. Smirnov, Proving AGT conjecture as HS duality: extension to five dimensions, 1105.0948.

[53] M. Blau and G. Thompson, Lectures on 2-d gauge theories: Topological aspects and path integral techniques, hep-th/9310144.

[54] F. Dolan, V. Spiridonov, and G. Vartanov, From 4d superconformal indices to $3 d$ partition functions, 1104.1787. 
[55] A. Gadde and W. Yan, Reducing the 4d Index to the $S^{3}$ Partition Function, 1104.2592.

[56] Y. Imamura, Relation between the $4 d$ superconformal index and the $S^{3}$ partition function, 1104.4482 .

[57] F. Benini, T. Nishioka, and M. Yamazaki, 4d Index to 3d Index and 2d TQFT, 1109.0283.

[58] I. Cherednik, Double affine Hecke algebras. London Mathematical Society lecture note series. Cambridge University Press, 2005.

[59] A. A. Kirillov Jr, Lectures on affine hecke algebras and macdonald's conjectures, Bull. Amer. Math. Soc. 34 (1997) 251-293.

[60] P. C. Argyres and J. R. Wittig, Infinite coupling duals of N=2 gauge theories and new rank 1 superconformal field theories, JHEP 01 (2008) 074, [0712.2028].

[61] F. Benini, S. Benvenuti, and Y. Tachikawa, Webs of five-branes and N=2 superconformal field theories, JHEP 09 (2009) 052, [arXiv:0906.0359].

[62] V. Spiridonov and G. Vartanov, Superconformal indices of N=4 SYM field theories, 1005.4196.

[63] P. I. Etingof and A. A. Kirillov, Jr., On the affine analog of Jack's and MacDonald's polynomials, hep-th/9403168.

[64] E. M. Rains, Transformations of Elliptic Hypergeometric Integrals, math/0309252.

[65] E. M. Rains, $B C_{n}$-symmetric abelian functions, math/0402113.

[66] A. Gorsky and N. Nekrasov, Hamiltonian systems of Calogero type and two-dimensional Yang-Mills theory, Nucl.Phys. B414 (1994) 213-238, [hep-th/9304047].

[67] J. A. Minahan and A. P. Polychronakos, Interacting fermion systems from two-dimensional QCD, Phys.Lett. B326 (1994) 288-294, [hep-th/9309044].

[68] A. Gorsky and N. Nekrasov, Relativistic Calogero-Moser model as gauged WZW theory, Nucl.Phys. B436 (1995) 582-608, [hep-th/9401017].

[69] A. Gorsky and N. Nekrasov, Elliptic Calogero-Moser system from two-dimensional current algebra, hep-th/9401021.

[70] A. Gorsky, Integrable many-body systems in the field theories, Theoretical and Mathematical Physics 103 (1995) 681-700. 10.1007/BF02065867.

[71] A. Gorsky and A. Mironov, Integrable many body systems and gauge theories, hep-th/0011197.

[72] A. A. Gerasimov and S. L. Shatashvili, Higgs Bundles, Gauge Theories and Quantum Groups, Commun.Math.Phys. 277 (2008) 323-367, [hep-th/0609024].

[73] S. Ruijsenaars, Elliptic integrable systems of Calogero-Moser type: A survey, Rokko Lectures in Math., no. 18 (2004) 201-221.

[74] N. A. Nekrasov and S. L. Shatashvili, Quantization of Integrable Systems and Four Dimensional Gauge Theories, 0908.4052. 
[75] N. Nekrasov and E. Witten, The Omega Deformation, Branes, Integrability, and Liouville Theory, JHEP 09 (2010) 092, [1002.0888].

[76] V. Spiridonov, Elliptic hypergeometric functions and calogero-sutherland-type models, Theoretical and Mathematical Physics 150 (2007) 266-277. 10.1007/s11232-007-0020-5.

[77] V. Spiridonov, Elliptic beta integrals and solvable models of statistical mechanics, 1011.3798.

[78] T. Nishioka, Y. Tachikawa, and M. Yamazaki, 3d Partition Function as Overlap of Wavefunctions, JHEP 1108 (2011) 003, [1105.4390].

[79] S. Benvenuti and S. Pasquetti, 3D-partition functions on the sphere: exact evaluation and mirror symmetry, 1105.2551.

[80] V. Dobrev and V. Petkova, All Positive Energy Unitary Irreducible Representations of Extended Conformal Supersymmetry, Phys.Lett. B162 (1985) 127-132.

[81] V. Dobrev and V. Petkova, Group Theoretical Approach to Extended Conformal Supersymmetry: Function Space Realizations and Invariant Differential Operators, Fortsch.Phys. 35 (1987) 537.

[82] F. A. Dolan and H. Osborn, On short and semi-short representations for four dimensional superconformal symmetry, Ann. Phys. 307 (2003) 41-89, [hep-th/0209056]. 